Geom. Funct. Anal. Vol. 19 (2009) 910-942

DOI $10.1007 / \mathrm{s} 00039-009-0019-1$

Published online October 22, 2009

GAFA Geometric And Functional Analysis

(C) 2009 The Author(s)

This article is published with open access at Springerlink.com

\title{
EXISTENCE AND UNIQUENESS OF CONSTANT MEAN CURVATURE FOLIATION OF ASYMPTOTICALLY HYPERBOLIC 3-MANIFOLDS
}

\author{
André Neves And Gang Tian
}

\begin{abstract}
We prove existence and uniqueness of foliations by stable spheres with constant mean curvature for 3-manifolds which are asymptotic to anti-de SitterSchwarzschild metrics with positive mass. These metrics arise naturally as spacelike timeslices for solutions of the Einstein equation with a negative cosmological constant.
\end{abstract}

\section{Introduction}

In 1996, Huisken and Yau [HuY] showed that metrics that arise as spacelike timeslices of solutions to the Einstein vacuum equation have a well-defined center of mass. More precisely, they showed that metrics which are asymptotic to Schwarzschild metrics with positive mass outside a compact set admit, under some technical assumptions, a unique foliation by stable spheres with constant mean curvature. We prove an analogous result for a large class of metrics that arise naturally as spacelike timeslices for solutions to the Einstein equation with negative cosmological constant. More precisely, we show that metrics which are, outside a compact set, asymptotic to Anti-de Sitter-Schwarzschild metrics with positive mass admit, under some technical assumptions, a unique foliation by stable spheres with constant mean curvature and thus, they have a well-defined center of mass.

Most of the work in this paper is devoted to proving uniqueness of foliations by stable spheres with constant mean curvature for asymptotically anti-de SitterSchwarzschild metrics with positive mass. Note that the uniqueness of such foliations fails for the hyperbolic 3-space and so the positivity of the mass needs to be used. The central idea in the paper consists in combining the positivity of the mass with the Kazdan-Warner obstructions $[\mathrm{K}]$ in order to prove uniqueness. The relation between positive mass and the study of foliations near infinity for asymptotically flat manifolds was observed by Christodoulou and Yau in [ChY].

The existence of constant mean curvature foliations in the asymptotically flat setting was proven by Huisken and Yau $[\mathrm{HuY}]$ using a volume preserving mean curvature flow and by $\mathrm{Ye}[\mathrm{Y}]$ using the implicit function theorem. For metrics asymptotic to Anti-de Sitter-Schwarzschild metrics, the existence of such foliations 
was proven by Rigger $[R]$ using the volume preserving mean curvature flow approach. In this setting, the method used in $[\mathrm{Y}]$ can also be adapted straightforwardly in order to obtain existence of foliations by constant mean curvature spheres. We include its proof in the last section for the sake completeness.

Before stating the main result we need to introduce some notation. Denote by $g_{0}$ the standard round metric on $S^{2}$. The anti-de Sitter-Schwarzschild manifold with mass $m>0$ is defined to be $\left(s_{0}, \infty\right) \times S^{2}$ with the metric

$$
g_{m}=\left(1+s^{2}-m / s\right)^{-1} d s^{2}+s^{2} g_{0},
$$

where $s_{0}$ is the zero of $1+s^{2}-m / s$. Note that when $m=0$ the anti-de SitterSchwarzschild metric becomes the hyperbolic metric. After a change of coordinates, the metric can be written in the form

$$
g_{m}=d r^{2}+\left(\sinh ^{2} r+m /(3 \sinh r)+O(\exp (-3 r))\right) g_{0} .
$$

Our result will apply to metrics which are, outside a compact set, lower order perturbations of Anti-de Sitter-Schwarzschild metrics.

DeFinition 1.1. $(M, g)$ is an asymptotically anti-de Sitter-Schwarzschild manifold with mass $m$ if, for some compact set $K, M-K$ is diffeomorphic to $\mathbb{R}^{3}$ minus a ball and, with respect to this diffeomorphism, the metric can be written (in spherical coordinates) as

$$
g=d r^{2}+\left(\sinh ^{2} r+m /(3 \sinh r)\right) g_{0}+Q
$$

where

$$
|Q|+|\nabla Q|+\left|\nabla^{2} Q\right|=O(\exp (-5 r)) .
$$

REMARK 1.2. (i) We point out that, even if it does not appear immediate, the Antide Sitter-Schwarzschild metrics $g_{m}$ satisfy the definition above. The reason is that the term $\sinh ^{2} r g_{0}$ has order $O(1)$ and so the term $O(\exp (-3 r)) g_{0}$ in the expression of $g_{m}$ has order $O(\exp (-5 r))$. Thus, it can be incorporated in the $Q$-term in the definition above.

(ii) A direct computation shows that the mass $m$ coincides with the mass defined in $[\mathrm{ChrH}]$ and $[\mathrm{W}]$ for asymptotically hyperbolic metrics.

With respect to the coordinates specified in the Definition 1.1, $M-K$ becomes equipped with a radial function $r$. Hence, given a foliation $\left(\Sigma_{t}\right)_{t \geq 0}$ of $M$, we can define the lower radius and the upper radius to be

$$
\underline{r}_{t}=\sup \left\{r(x) \mid x \in \Sigma_{t}\right\} \quad \text { and } \quad \bar{r}_{t}=\inf \left\{r(x) \mid x \in \Sigma_{t}\right\}
$$

respectively. Given a family of functions $f_{t}$ defined on $\Sigma_{t}$, we use

$$
f_{t}=O\left(\exp \left(-n \underline{r}_{t}\right)\right) \text { and } f_{t}=o\left(\exp \left(-n \underline{r}_{t}\right)\right)
$$

to denote that

$$
\limsup _{t \rightarrow \infty}\left(\left|f_{t}\right| \exp \left(n \underline{r}_{t}\right)\right)<\infty \quad \text { and } \quad \limsup _{t \rightarrow \infty}\left(\left|f_{t}\right| \exp \left(n \underline{r}_{t}\right)\right)=0
$$

respectively.

A surface $\Sigma$ with constant mean curvature is said to be stable if volume preserving variations do not decrease its area. A standard computation shows that stability is equivalent to the second variation operator

$$
L u \equiv-\Delta f-\left(|A|^{2}+R(\nu, \nu)\right) f
$$


having only nonnegative eigenvalues when restricted to functions with zero mean value, i.e.

$$
\int_{\Sigma_{t}}\left(|A|^{2}+R(\nu, \nu)\right) f^{2} d \mu \leq \int_{\Sigma_{t}}|\nabla f|^{2} d \mu
$$

for all functions $f$ with $\int_{\Sigma_{t}} f d \mu=0$.

The purpose of this paper is to show the following result.

Theorem 1.3. Asymptotically anti-de Sitter-Schwarzschild manifolds with positive mass admit a unique foliation $\left(\Sigma_{t}\right)_{t \geq 0}$ by stable spheres with constant mean curvature such that

$$
\lim _{t \rightarrow \infty}\left(\bar{r}_{t}-6 / 5 \underline{r}_{t}\right)=-\infty
$$

REMARK 1.4. A condition similar to (1) was also assumed in $[\mathrm{HuY}]$ for metrics that are asymptotic Schwarzschild metrics. It is an interesting question if one can strengthen Theorem 1.3 by weakening condition (1) to the condition that $\underline{r}_{t}$ is sufficiently large. Its analogous version for metrics asymptotic to Schwarzschild metrics was solved in $[\mathrm{QT}]$.

A contradiction argument implies the following corollary.

Corollary 1.5. There are positive constants $C_{0}$ and $R_{0}$ depending only on $g$ so that any stable sphere $\Sigma$ with constant mean curvature satisfying

$$
|\Sigma| \geq C_{0} \quad \text { and } \quad \bar{r}-6 / 5 \underline{r} \leq-R_{0}
$$

is unique.

Proof. Assume there are two distinct sequences $\left(\Sigma_{i}^{1}\right)_{i \in \mathbb{N}}$ and $\left(\Sigma_{i}^{2}\right)_{i \in \mathbb{N}}$ of stable spheres with constant mean curvature such that

$$
\begin{gathered}
\lim _{i \rightarrow \infty}\left|\Sigma_{i}^{1}\right|=\lim _{i \rightarrow \infty}\left|\Sigma_{i}^{2}\right|=\infty, \\
\lim _{i \rightarrow \infty} \bar{r}_{i}^{1}-6 / 5 \underline{r}_{i}^{1}=\lim _{i \rightarrow \infty} \bar{r}_{i}^{2}-6 / 5 \underline{r}_{i}^{2}=-\infty,
\end{gathered}
$$

and $H\left(\Sigma_{i}^{1}\right)=H\left(\Sigma_{i}^{2}\right)$ for every integer $i$. The proof of Theorem 1.3 also applies to the foliations

$$
\Sigma_{t}^{j} \equiv \Sigma_{i}^{j} \quad \text { if } i \leq t<i+1,
$$

where $j=1,2$, and so we obtain a contradiction.

Acknowledgement. The first author would like to express his gratitude to Alice Chang for many useful discussions.

\section{Outline of the Proof}

We outline the proof of Theorem 1.3 in order to emphasize the main ideas over the technical aspects of the paper. Let $\left(\Sigma_{t}\right)_{t \geq 0}$ denote a foliation by stable spheres with constant mean curvature. For the sake of simplicity, we assume in this discussion that $\bar{r}_{t}-\underline{r}_{t}$ is uniformly bounded. We use the notation

$$
f_{\Sigma_{t}} f d \mu \equiv\left|\Sigma_{t}\right|^{-1} \int_{\Sigma_{t}} f d \mu,
$$

where $\left|\Sigma_{t}\right|$ stands for the surface area of $\Sigma_{t}$. 
Section 3 is devoted to auxiliary computations. In section 4 we follow the same argumentation done by Huisken and Yau in $[\mathrm{HuY}]$ and use the stability assumption in order to derive the following estimate for the mean curvature of $\Sigma_{t}$ (Lemma 4.1)

$$
H^{2}=4+16 \pi /\left|\Sigma_{t}\right|+f_{\Sigma_{t}} O(\exp (-3 r)) d \mu
$$

and the following integral estimate for the trace free part of the second fundamental form (Proposition 4.3)

$$
f_{\Sigma_{t}}|\AA|^{2} d \mu \leq O\left(\exp \left(-4 \underline{r}_{t}\right)\right) .
$$

In section 5 we study the intrinsic geometry of $\Sigma_{t}$. More precisely, we show that after pulling back by a suitable diffeomorphism from $\Sigma_{t}$ to $S^{2}$, the metric

$$
\hat{g}_{t} \equiv 4 \pi /\left|\Sigma_{t}\right| g
$$

can be written as

$$
\exp \left(2 \beta_{t}\right) g_{0}
$$

where $g_{0}$ denotes the standard round metric on $S^{2}$ and

$$
\beta_{t}=O\left(\exp \left(-\underline{r}_{t}\right)\right) \text {. }
$$

This result implies that $\hat{g}_{t}$ is very "close" to being a round metric. The proof of this result (Theorem 5.1) has two steps. The first step consists in deriving a pointwise estimate for $|\stackrel{A}{\mid}|$ from the integral estimate (Proposition 5.3). In order to do so, we have to exploit the fact that the hyperbolic metric is conformal to the Euclidean metric on the unit ball and so the same is true, up to a term of low order, for the metric $g$. Therefore, denoting by $d \sigma$ the surface measure induced by the Euclidean metric on $\Sigma_{t}$, we have by conformal invariance that

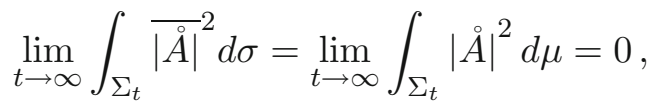

where the quantities measured with respect to the Euclidean metric are denoted by a bar. Because the Euclidean area of $\Sigma_{t}$ converges to $4 \pi$ (Proposition 4.2), the identity above and the Gauss-Bonnet theorem imply that the Euclidean mean curvature $\bar{H}$ has no concentration points. We can then use Michael-Simon Sobolev inequality and the equation satisfied by $|\stackrel{\AA}{\mid}|^{2}$ (with respect to the Euclidean metric) in order to apply the standard Moser iteration procedure and conclude that, up to lower-order terms,

$$
\sup _{\Sigma_{t}}|\AA|^{2} \leq C \int_{\Sigma_{t}}|\AA|^{2} d \sigma=C \int_{\Sigma_{t}}|\AA|^{2} d \mu \leq O\left(\exp \left(-4 \underline{r}_{t}\right)\right) .
$$

The second step consists in using the Gauss equation (Lemma 3.2) which, combined with estimates (2) and (3), implies that the Gaussian curvature of $\Sigma_{t}$ with respect to $\hat{g}_{t}$ satisfies

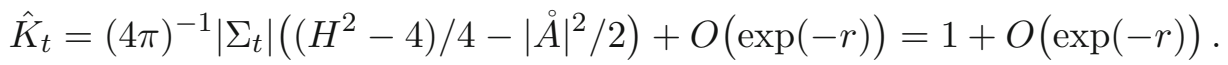

The desired result follows from this estimate.

Section 6 contains the main estimate that makes uniqueness possible. Set

$$
w_{t}(x) \equiv r(x)-\hat{r}_{t}, \quad \text { where } \quad\left|\Sigma_{t}\right|=4 \pi \sinh ^{2} \hat{r}_{t} .
$$


We want to show that $w_{t}$ converges uniformly to zero (Theorem 6.1 ). With respect to the standard round metric $g_{0}$, the functions $w_{t}$ satisfy the equation (see (14))

$$
\Delta_{0} w_{t}=\exp \left(-2 w_{t}\right)-1+P,
$$

where

$$
\int_{S^{2}}|P| d \mu_{0}=O(\exp (-r)) \text {. }
$$

Because we are assuming that $\bar{r}_{t}-\underline{r}_{t}$ is uniformly bounded, we have that $w_{t}$ is bounded in $W^{1,2}$ with respect to $g_{0}$ and thus, we can take a sequence converging weakly to $w_{0}$ that satisfies

$$
\Delta_{0} w_{0}=\exp \left(-2 w_{0}\right)-1 .
$$

It is well known that this equation can have many solutions. Therefore, we need to use the fact that the mass is nonzero in order to show that $w_{0}=0$. This is achieved through the Kazdan-Warner identity $[\mathrm{K}]$. Because the metric $\hat{g}_{t}$ is "close" to being the round metric, this identity implies that, for each of the standard coordinate functions $x_{1}, x_{2}, x_{3}$ on $S^{2}$ (see (16)),

$$
\int_{S^{2}} x_{i} \hat{K}_{t} d \mu_{0}=O\left(\exp \left(-2 \underline{r}_{t}\right)\right), \quad \text { for } i=1,2,3 .
$$

On the other hand, a careful expansion of the terms involved in the Gauss equation shows that the Gaussian curvature of $\hat{g}_{t}$ is such that

$$
\left|\Sigma_{t}\right|^{1 / 2} \hat{K}_{t}=(4 \pi)^{-1}\left|\Sigma_{t}\right|^{3 / 2}\left(H^{2}-4\right) / 4+m(4 \pi)^{-1}\left|\Sigma_{t}\right|^{3 / 2} / \sinh ^{3} r+O(\exp (-r)) \text {. }
$$

Hence, because the mean curvature is constant, we obtain from the Kazdan-Warner identity that (Proposition 6.3)

Therefore,

$$
m \int_{S^{2}} x_{i} \exp \left(-3 w_{t}\right) d \mu_{0}=O\left(\exp \left(-\underline{r}_{t}\right)\right), \quad \text { for } i=1,2,3 .
$$

$$
m \int_{S^{2}} x_{i} \exp \left(-3 w_{0}\right) d \mu_{0}=0, \quad \text { for } i=1,2,3 .
$$

Recalling that $w_{0}$ is a solution to equation (4), the above identity implies that $w_{0}=0$. Standard techniques can then be used to show that $w_{t}$ converges to zero uniformly.

In section 7 we improve the rate of convergence of $w_{t}$ to zero (Theorem 7.1 ). In order to do so, we redefine $w_{t}$ to be

$$
w_{t}(x) \equiv r(x)-\tilde{r}_{t},
$$

where $\tilde{r}_{t}$ is such that

$$
H=2 \cosh \tilde{r}_{t} / \sinh \tilde{r}_{t}-m / \sinh ^{3} \tilde{r}_{t}+o\left(\exp \left(-4 \tilde{r}_{t}\right)\right) .
$$

Then, the equation satisfied by $w_{t}$ improves to become

$$
\Delta_{0} w_{t}=\exp \left(-2 w_{t}\right)-1+o(\exp (-2 r)) \text {. }
$$

Using the orthogonality condition given by the Kazdan-Warner obstructions, standard elliptic estimates show that

$$
\left|w_{t}\right|_{C^{2, \alpha}}=o\left(\exp \left(-\underline{r}_{t}\right)\right) .
$$

In section 8.1, we use the strong approximation of $\Sigma_{t}$ to a coordinate sphere in order to prove uniqueness of foliations by stable spheres with constant mean 
curvature. The main reason for this to work is that, with respect to the round metric on $S^{2}$, the linearization of the mean curvature on a coordinate sphere $\{|x|=r\}$ is the operator

$$
L(f)=\Delta_{0} f+(2-3 m / \sinh r) f,
$$

which is invertible if $m$ is not zero. Finally, in section 8.2, we adapt the arguments used in $[\mathrm{Y}]$ and we show that, for all $r$ sufficiently large, we can find a stable sphere with constant mean curvature which is a perturbation of $\{|x|=r\}$.

\section{Preliminaries}

In this section we compute the relevant formulas needed throughout this paper. Before doing so, we need to introduce some notation. $\Sigma$ is assumed to be a stable sphere with constant mean curvature. The radial vector is denoted by $\partial_{r}$, and $\partial_{r}^{\top}$ stands for the tangential projection of $\partial_{r}$ on $T \Sigma$, which has length denoted by $\left|\partial_{r}^{\top}\right|$. Finally, $\nu$ denotes the exterior unit normal to $\Sigma$.

We start by computing the asymptotic expansion of some geometric quantities depending on $g_{m}$, the Anti de Sitter- Schwarzschild metric with mass $m$. Let $\left\{e_{1}, e_{2}\right\}$ denote a $g_{m}$-orthonormal basis for the coordinate spheres $\{|x|=r\}$.

Lemma 3.1. (i) The mean curvature $H_{m}(r)$ of $\{|x|=r\}$ is such that

$$
H_{m}(r)=2 \cosh r / \sinh r-m / \sinh ^{3} r+O(\exp (-5 r)) \text {. }
$$

(ii) The scalar curvature is -6 .

(iii) The Ricci curvature is such that

$$
\begin{aligned}
& R_{m}\left(\partial_{r}, \partial_{r}\right)=-2-m / \sinh ^{3} r+O(\exp (-5 r)) \\
& R_{m}\left(e_{1}, e_{1}\right)=R_{m}\left(e_{2}, e_{2}\right)=-2+m /\left(2 \sinh ^{3} r\right)+O(\exp (-5 r)) \\
& R_{m}\left(e_{1}, e_{2}\right)=0 .
\end{aligned}
$$

(iv) The derivatives of the Ricci curvature with respect $g_{m}$ are such that

$$
\nabla_{e_{1}}^{m} R_{m}\left(\partial_{r}, e_{1}\right)=\nabla_{e_{2}}^{m} R_{m}\left(\partial_{r}, e_{2}\right) .
$$

Proof. If we write $g_{m}$ as $d r^{2}+\psi^{2}(r) g_{0}$, then

$$
H_{m}(r)=2 \psi^{\prime}(r) / \psi(r),
$$

and so the first formula follows. The second formula is just a direct computation. The rotational symmetry of the metric implies that its Gaussian curvature is $\psi^{-2}(r)$ and that the second fundamental form of $\{|x|=r\}$ is trace free. Hence, we have from Gauss equation that

$$
R_{m}\left(\partial_{r}, \partial_{r}\right)=-3-\psi^{-2}(r)+H^{2} / 4
$$

and so the first identity in (iii) follows. The other two identities in (iii) are a consequence of (ii) and rotational symmetry. The last identity follows from the same type of arguments.

The next lemma relates the mass $m$ of a metric $g$ with the Gaussian curvature $K$ of a surface $\Sigma$. 
LEMma 3.2. The Gaussian curvature of $\Sigma$ satisfies

$$
K=\left(H^{2}-4\right) / 4+m / \sinh ^{3} r-3 m\left|\partial_{r}^{\top}\right|^{2} /\left(2 \sinh ^{3} r\right)-|\AA|^{2} / 2+O(\exp (-5 r)) .
$$

Proof. Because $g$ is $C^{2}$-perturbation of order $O(\exp (5 r))$ of $g_{m}$, we have

$$
R=-6+O(\exp (-5 r))
$$

and

$$
\begin{aligned}
2+R(\nu, \nu) & =2+R_{m}(\nu, \nu)+O(\exp (-5 r)) \\
& =-m / \sinh ^{3} r+3 m\left|\partial_{r}^{\top}\right|^{2} /\left(2 \sinh ^{3} r\right)+O(\exp (-5 r)) .
\end{aligned}
$$

The result follows from Gauss equation

$$
K=R / 2-R(\nu, \nu)+H^{2} / 4-|\AA|^{2} / 2 .
$$

Next, we derive the equation satisfied by the trace-free part of the second fundamental form $\AA$.

Lemma 3.3. The Laplacian of $|\AA|^{2}$ satisfies

$$
\begin{aligned}
\Delta\left(|\AA|^{2} / 2\right)=\left(\frac{H^{2}-4}{2}\right. & \left.-|\AA|^{2}\right)|\AA|^{2}+|\nabla \AA|^{2} \\
& +\left(|\AA|^{2}+|H|\left|\partial_{r}^{\top}\right|^{2}|\AA|\right) O(\exp (-3 r))+|\AA| O(\exp (-5 r)) .
\end{aligned}
$$

Proof. We assume normal coordinates $x=\left\{x^{i}\right\}_{i=1,2}$ around a point $p$ in the constant mean curvature surface $\Sigma$. The tangent vectors are denoted by $\left\{\partial_{1}, \partial_{2}\right\}$, the normal vector by $\nu$, and the Einstein summation convention for the sum of repeated indices is used.

Simons' identity for the Laplacian of the second fundamental form $A$ (see for instance $[\mathrm{HuP}])$ implies that

$$
\begin{aligned}
\Delta\left(|\AA|^{2} / 2\right)=|\nabla \AA|^{2} & +H \operatorname{Tr}\left(\AA^{3}\right)+H^{2}|\AA|^{2} / 2-|\AA|^{4} \\
+ & H \AA_{i j} R_{\nu i \nu j}-R_{\nu \nu}|\AA|^{2}+2 R_{k i k m} \AA_{m j} \AA_{i j} \\
& +2 R_{k i j m} \AA_{k m} \AA_{i j}+\AA_{i j}\left(\nabla_{k} R_{\nu j i k}+\nabla_{i} R_{\nu k j k}\right) .
\end{aligned}
$$

From

if follows that

$$
R_{s t u v}=-\left(\delta_{s u} \delta_{t v}-\delta_{s v} \delta_{t u}\right)+O(\exp (-3 r))
$$

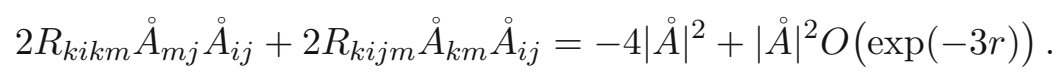

Hence, $\operatorname{Tr}\left(\AA^{3}\right)=0$ implies that

$$
\begin{aligned}
\Delta\left(|\AA|^{2} / 2\right)=|\nabla \AA|^{2}+\left(\frac{H^{2}-4}{2}\right. & \left.-|\AA|^{2}\right)|\AA|^{2}+H R_{\nu i \nu j} \AA_{i j} \\
& +\left(\nabla_{k} R_{\nu j i k}+\nabla_{i} R_{\nu k j k}\right) \AA_{i j}+|\AA|^{2} O(\exp (-3 r)) .
\end{aligned}
$$

On the other hand, if $\left\{v_{1}, v_{2}\right\}$ is an eigenbasis for $\AA$, we obtain from Lemma 3.1 that

$$
\begin{aligned}
R_{\nu i \nu j} \stackrel{\AA}{i j}_{i j} & =\frac{|\stackrel{\AA}{ }|}{\sqrt{2}}\left(R\left(v_{1}, v_{1}\right)-R\left(v_{2}, v_{2}\right)\right) \\
& =\frac{\mid \AA ्}{\sqrt{2}}\left(R_{m}\left(v_{1}, v_{1}\right)-R_{m}\left(v_{2}, v_{2}\right)\right)+|\AA| O(\exp (-5 r))
\end{aligned}
$$




$$
=\left|\partial_{r}^{\top}\right|^{2}|\AA| O(\exp (-3 r))+|\AA| O(\exp (-5 r))
$$

and

$$
\begin{aligned}
\left(\nabla_{k} R_{\nu j i k}+\nabla_{i} R_{\nu k j k}\right) \AA_{i j} & =\sqrt{2}|\stackrel{\AA}{ }|\left(\nabla_{v_{1}} R\left(\nu, v_{1}\right)-\nabla_{v_{2}} R\left(\nu, v_{2}\right)\right) \\
& =\left|\partial_{r}^{\top}\right|^{2}|\AA| O(\exp (-3 r))+|\AA| O(\exp (-5 r)) .
\end{aligned}
$$

Thus, the desired result follows.

Finally, we derive the equation satisfied by the Laplacian of $r$ on $\Sigma$. Throughout the rest of this paper, we will progressively estimate and explore all of its terms.

Proposition 3.4. The Laplacian of $r$ on $\Sigma$ satisfies

$$
\begin{aligned}
\Delta r=\left(4-2\left|\partial_{r}^{\top}\right|^{2}\right) \exp ( & -2 r)+2-H \\
+ & (H-2)\left(1-\left\langle\partial_{r}, \nu\right\rangle\right)+\left(1-\left\langle\partial_{r}, \nu\right\rangle\right)^{2}+O(\exp (-3 r))
\end{aligned}
$$

or, being more detailed,

$$
\begin{aligned}
\Delta r=2 \cosh r / \sinh r-m / \sinh ^{3} r-H & \\
+(H-2)\left(1-\left\langle\partial_{r}, \nu\right\rangle\right)+(1- & \left.\left\langle\partial_{r}, \nu\right\rangle\right)^{2}-2\left|\partial_{r}^{\top}\right|^{2} \exp (-2 r) \\
& +\left|\partial_{r}^{\top}\right|^{2} O(\exp (-3 r))+O(\exp (-5 r)) .
\end{aligned}
$$

Proof. It suffices to prove the second identity. Using again normal coordinates $\left\{x_{1}, x_{2}\right\}$, we have from Lemma 3.1

$$
\begin{aligned}
\operatorname{div}_{\Sigma} \partial_{r}= & \left\langle\nabla_{i}^{m} \partial_{r}, \partial_{i}\right\rangle+O(\exp (-5 r)) \\
= & H^{m}(r)-H^{m}(r)\left|\partial_{r}^{\top}\right|^{2} / 2+O(\exp (-5 r)) \\
= & \left(2-\left|\partial_{r}^{\top}\right|^{2}\right) \cosh r / \sinh r-m / \sinh ^{3} r \\
& \quad+\left|\partial_{r}^{\top}\right|^{2} O(\exp (-3 r))+O(\exp (-5 r)) .
\end{aligned}
$$

On the other hand,

$$
\operatorname{div}_{\Sigma} \partial_{r}=\Delta r+\left\langle\partial_{r}, \nu\right\rangle H
$$

and the result follows from the easily checked identity

$$
\begin{aligned}
\left|\partial_{r}^{\top}\right|^{2} \cosh r / \sinh r+ & \left\langle\partial_{r}, \nu\right\rangle H=H-(H-2)\left(1-\left\langle\partial_{r}, \nu\right\rangle\right) \\
& -\left(1-\left\langle\partial_{r}, \nu\right\rangle\right)^{2}+2\left|\partial_{r}^{\top}\right|^{2} \exp (-2 r)+\left|\partial_{r}^{\top}\right|^{2} O(\exp (-4 r)) .
\end{aligned}
$$

\section{Integral Estimates}

We use the stability condition in the same spirit as in $[\mathrm{HuY}]$ in order to estimate the mean curvature $H$ and the $L^{2}$ norm of $|\AA|$. In the next section, we combine these integral estimates with Lemma 3.3 in order to obtain pointwise estimates for $|\AA|$. From this section on,

$$
\left(\Sigma_{t}\right)_{t \geq 0}
$$

denotes a foliation by stable spheres with constant mean curvature satisfying condition (1). We omit the index $t$ in the notation whenever it becomes obvious that we are referring to quantities depending on $\Sigma_{t}$. 
Lemma 4.1. For each $t$, we have that

or, equivalently,

$$
H^{2}=4+16 \pi /\left|\Sigma_{t}\right|+f_{\Sigma_{t}} O(\exp (-3 r)) d \mu,
$$

$$
H=2+4 \pi /\left|\Sigma_{t}\right|+f_{\Sigma_{t}} O(\exp (-3 r)) d \mu .
$$

Proof. The stability condition implies that, after a clever choice of test function (see [HuY, Prop. 5.3]),

$$
8 \pi \geq \int_{\Sigma_{t}}|A|^{2}+R(\nu, \nu) d \mu=\int_{\Sigma_{t}}|\AA|^{2}+\left(H^{2}-4\right) / 2+R(\nu, \nu)+2 d \mu
$$

and so, because

$$
R(\nu, \nu)=-2+O(\exp (-3 r))
$$

we have

$$
H^{2} \leq 4+16 \pi /\left|\Sigma_{t}\right|+f_{\Sigma_{t}} O(\exp (-3 r)) d \mu .
$$

On the other hand, from Lemma 3.2 and the Gauss-Bonnet theorem we obtain that

$$
H^{2}=4+16 \pi /|\Sigma|+2 f_{\Sigma_{t}}|\AA|^{2} d \mu+f_{\Sigma_{t}} O(\exp (-3 r)) d \mu,
$$

and thus the result follows.

This lemma combined with the equation for $\Delta r$ gives us these first estimates regarding $\Sigma_{t}$.

Proposition 4.2. The following identities hold:

(i)

$$
\int_{\Sigma_{t}} \exp (-2 r) d \mu=\pi+O\left(\exp \left(-\underline{r}_{t}\right)\right) .
$$

In particular, there is a constant $C$ so that

$$
C^{-1} \exp \left(2 \underline{r}_{t}\right) \leq\left|\Sigma_{t}\right| \leq C \exp \left(2 \bar{r}_{t}\right)
$$

(ii)

$$
\int_{\Sigma_{t}}\left(1-\left\langle\partial_{r}, \nu\right\rangle\right)^{2} d \mu=O\left(\exp \left(-\underline{r}_{t}\right)\right)
$$

(iii) For every positive integer $j$

$$
\int_{\Sigma_{t}} j\left|\partial_{r}^{\top}\right|^{2} \exp (-j r) d \mu=O\left(\exp \left(-j \underline{r}_{t}\right)\right) .
$$

Proof. Integrating the first identity in Proposition 3.4 and using Lemma 4.1 we obtain

$$
\begin{aligned}
4 \pi+\int_{\Sigma_{t}} O(\exp (-3 r)) d \mu= & \int_{\Sigma_{t}}\left(4-2\left|\partial_{r}^{\top}\right|^{2}\right) \exp (-2 r) d \mu \\
& +f_{\Sigma_{t}} 4 \pi\left(1-\left\langle\partial_{r}, \nu\right\rangle\right) d \mu+\int_{\Sigma_{t}}\left(1-\left\langle\partial_{r}, \nu\right\rangle\right)^{2} d \mu .
\end{aligned}
$$

Hence, both quantities in (i) and (ii) are uniformly bounded because

$$
\int_{\Sigma_{t}} O(\exp (-3 r)) d \mu=O\left(\exp \left(2 \bar{r}_{t}-3 \underline{r}_{t}\right)\right)
$$


Integrating the identity

$$
\Delta(\exp (-j r) / j)=-\exp (-j r) \Delta r+j \exp (-j r)\left|\partial_{r}^{\top}\right|^{2}
$$

we obtain, after using Lemma 4.1 and Proposition 3.4,

$$
\begin{aligned}
\int_{\Sigma_{t}} j\left|\partial_{r}^{\top}\right|^{2} & \exp (-j r) d \mu=\int_{\Sigma_{t}}\left(4-2\left|\partial_{r}^{\top}\right|^{2}\right) \exp (-(j+2) r) d \mu \\
& -4 \pi f_{\Sigma_{t}} \exp (-j r) d \mu+f_{\Sigma_{t}} 4 \pi\left(1-\left\langle\partial_{r}, \nu\right\rangle\right) \exp (-j r) d \mu \\
+ & \int_{\Sigma_{t}}\left(1-\left\langle\partial_{r}, \nu\right\rangle\right)^{2} \exp (-j r) d \mu+\int_{\Sigma_{t}} O(\exp (-(3+j) r)) d \mu \\
& +f_{\Sigma_{t}} O(\exp (-3 r)) d \mu \int_{\Sigma_{t}} O(\exp (-3 r)) d \mu .
\end{aligned}
$$

As a result, the third identity follows. Next, we use (iii) and the Euclidean isoperimetric identity to prove both (i) and (ii).

With respect to the unit ball model for the hyperbolic metric, the metric $g$ can be written as

$$
g=\psi^{2}\left(d x^{2}+d y^{2}+d z^{2}\right)+O\left(\psi^{-3}\right),
$$

where

$$
\psi(x) \equiv \frac{2}{1-|x|^{2}} \quad \text { and } \quad \sinh r=\frac{2|x|}{1-|x|^{2}} .
$$

If $\underline{s}_{t}$ denotes the radius of the largest Euclidean ball centered at the origin that is contained in the interior of $\Sigma_{t}$, then $\sinh \underline{r}_{t}=2 \underline{s}_{t} /\left(1-\underline{s}_{t}^{2}\right)$. The Euclidean isoperimetric inequality implies that

$$
\operatorname{area}\left(\Sigma_{t}\right) \geq(36 \pi)^{1 / 3} \operatorname{volume}\left(\left\{|x|=\underline{s}_{t}\right\}\right)^{2 / 3}=4 \pi \underline{s}_{t}^{2},
$$

where the area and volume are measured with respect to the Euclidean metric. Thus, denoting by $d \sigma$ the surface measure induced by the Euclidean metric, we have

$$
\begin{aligned}
\int_{\Sigma_{t}} 4 \exp (-2 r) d \mu & \geq \int_{\Sigma_{t}} \sinh ^{-2} r d \mu-\int_{\Sigma_{t}} \exp (-2 r) \sinh ^{-2} r d \mu \\
& =\int_{\Sigma_{t}}|x|^{-2} d \sigma+O\left(\exp \left(-2 \underline{r}_{t}\right)\right) \geq 4 \pi-4 \pi\left(1-\underline{s}^{2}\right)+O\left(\exp \left(-2 \underline{r}_{t}\right)\right) \\
& =4 \pi+O\left(\exp \left(-\underline{r}_{t}\right)\right)
\end{aligned}
$$

On the other hand,

$$
f_{\Sigma_{t}}\left(1-\left\langle\partial_{r}, \nu\right\rangle\right) d \mu \leq\left|\Sigma_{t}\right|^{-1 / 2}\left(\int_{\Sigma_{t}}\left(1-\left\langle\partial_{r}, \nu\right\rangle\right)^{2} d \mu\right)^{1 / 2}=O\left(\exp \left(-r_{t}\right)\right)
$$

and, recalling that both quantities in (i) and (ii) are uniformly bounded, we obtain from (5) that

$$
4 \pi+O\left(\exp \left(-\underline{r}_{t}\right)\right)=\int_{\Sigma_{t}} 4 \exp (-2 r) d \mu+\int_{\Sigma_{t}}\left(1-\left\langle\partial_{r}, \nu\right\rangle\right)^{2} d \mu .
$$

Therefore, (i) and (ii) follow from this estimate combined with (6).

Next, we use the stability of $\Sigma_{t}$ in the same way as in $[\mathrm{HuY}, \S 5]$ in order to obtain integral estimates for $\AA$. 
Proposition 4.3. The following estimate holds

$$
\begin{aligned}
f_{\Sigma_{t}}|\AA|^{2} d \mu+\int_{\Sigma_{t}}|\AA|^{4} d \mu+\int_{\Sigma_{t}}|\nabla \AA|^{2} d \mu \leq \int_{\Sigma_{t}}\left|\partial_{r}^{\top}\right|^{2} O(\exp (-4 r)) d \mu & +\int_{\Sigma_{t}} O(\exp (-7 r)) d \mu .
\end{aligned}
$$

In particular,

$$
f_{\Sigma_{t}}|\AA|^{2} d \mu \leq O\left(\exp \left(-4 \underline{r}_{t}\right)\right) .
$$

Proof. Integrating the identity in Lemma 3.3 and using Lemma 4.1 we obtain

$$
\begin{aligned}
8 \pi f_{\Sigma_{t}}|\AA|^{2} d \mu & +\int_{\Sigma_{t}}|\nabla \AA|^{2} d \mu=\int_{\Sigma_{t}}\left(|\AA|^{2}+|\AA|\left|\partial_{r}^{\top}\right|^{2}\right) O(\exp (-3 r)) d \mu \\
& +\int_{\Sigma_{t}}|\AA|^{4} d \mu+\int_{\Sigma_{t}}|\AA|^{2} d \mu \int_{\Sigma_{t}} O(\exp (-3 r)) d \mu+\int_{\Sigma_{t}}|\AA| O(\exp (-5 r)) d \mu .
\end{aligned}
$$

We now argue that, for every fixed $\varepsilon>0$,

$$
\begin{aligned}
(8 \pi+o(1)) f_{\Sigma_{t}}|\AA|^{2} d \mu & +\int_{\Sigma_{t}}|\nabla \AA|^{2} d \mu=(1+\varepsilon / 2) \int_{\Sigma_{t}}|\AA|^{4} d \mu \\
& +\int_{\Sigma_{t}}\left|\partial_{r}^{\top}\right|^{2} O(\exp (-4 r)) d \mu+\int_{\Sigma_{t}} O(\exp (-7 r)) d \mu .
\end{aligned}
$$

This is true because

$$
\begin{gathered}
\int_{\Sigma_{t}}|\AA| O(\exp (-5 r)) d \mu \leq \int_{\Sigma_{t}}|\AA|^{2} O\left(\exp \left(-3 \underline{r}_{t}\right)\right) d \mu+\int_{\Sigma_{t}} O(\exp (-7 r)) d \mu, \\
|\AA|\left|\partial_{r}^{\top}\right|^{2} O(\exp (-3 r)) \leq \varepsilon / 2|\AA|^{4}+\left|\partial_{r}^{\top}\right|^{2} O(\exp (-4 r)),
\end{gathered}
$$

and, due to Proposition 4.2,

$$
\lim _{t \rightarrow \infty} f_{\Sigma_{t}} O(\exp (-3 r)) d \mu=0
$$

Before we use the stability of $\Sigma_{t}$, let us first remark that, according to Lemma 3.1 and Lemma 4.1, we have for all $t$ sufficiently large

$$
|A|^{2}+R(\nu, \nu) \geq|\stackrel{\AA}{A}|^{2}+2+R(\nu, \nu) \geq|\AA|^{2}+O(\exp (-3 r)) .
$$

Hence, the stability assumption implies that

$$
\int_{\Sigma_{t}}|\stackrel{\AA}{A}|^{2} f^{2} d \mu \leq \int_{\Sigma_{t}}|\nabla f|^{2} d \mu+\int_{\Sigma_{t}} f^{2} O(\exp (-3 r)) d \mu
$$

for all functions $f$ with $\int_{\Sigma_{t}} f d \mu=0$. Using the test function (see [HuY, §5])

$$
f=u-f_{\Sigma_{t}} u d \mu \equiv u-\bar{u}, \quad \text { with } u=|\AA|
$$

we obtain

$$
\int_{\Sigma_{t}}|\AA|^{4} d \mu \leq\left.\int_{\Sigma_{t}}|\nabla| \AA\right|^{2} d \mu+2 \bar{u} \int_{\Sigma_{t}}|\AA|^{3} d \mu+\int_{\Sigma_{t}}(u-\bar{u})^{2} O(\exp (-3 r)) d \mu .
$$

Looking at the proof of Lemma 4.1 we see that

$$
\int_{\Sigma_{t}}|\stackrel{\AA}{A}|^{2} d \mu=\int_{\Sigma_{t}} O(\exp (-3 r)) d \mu=o(1)
$$


and so, because

$$
\bar{u}^{2} \leq f_{\Sigma_{t}}|\AA|^{2} d \mu,
$$

we obtain for every $\varepsilon>0$ fixed

$$
\begin{aligned}
\int_{\Sigma_{t}}|\AA|^{4} d \mu \leq \varepsilon \int_{\Sigma_{t}}|\AA|^{4} d \mu+o & (1) \int_{\Sigma_{t}}|\AA|^{2} d \mu \\
& +\left.\int_{\Sigma_{t}}|\nabla| \AA\right|^{2} d \mu+\int_{\Sigma_{t}}(u-\bar{u})^{2} O(\exp (-3 r)) d \mu .
\end{aligned}
$$

We now estimate the last two terms in this inequality. If $\left\{e_{1}, e_{2}\right\}$ denotes an eigenbasis for $\AA$, one can easily check that

$$
\left.|\nabla| \AA\right|^{2}=2\left|\nabla \AA\left(e_{1}, e_{1}\right)\right|^{2}, \quad \nabla \AA\left(e_{1}, e_{1}\right)=-\nabla \AA\left(e_{2}, e_{2}\right)
$$

and

$$
|\nabla \AA|^{2}=|\nabla| \AA||^{2}+2\left|\nabla \AA\left(e_{1}, e_{2}\right)\right|^{2} .
$$

Using Codazzi equations and Lemma 3.1 we have that, for $i, j=1,2$,

$$
\begin{aligned}
\nabla_{i} \AA\left(e_{i}, e_{j}\right) & =\nabla_{j} \AA\left(e_{i}, e_{i}\right)+R\left(\nu, e_{j}\right) \\
& =\nabla_{j} \AA\left(e_{i}, e_{i}\right)+\left|\partial_{r}^{\top}\right| O(\exp (-3 r))+O(\exp (-5 r)),
\end{aligned}
$$

and this implies that, for every $\varepsilon>0$,

$$
\left|\nabla_{i} \AA\left(e_{i}, e_{j}\right)\right|^{2} \geq(1-\varepsilon / 2)\left|\nabla_{j} \AA\left(e_{i}, e_{i}\right)\right|^{2}+\left|\partial_{r}^{\top}\right|^{2} O(\exp (-6 r))+O(\exp (-10 r)) \text {. }
$$

Therefore, we can estimate

$$
2\left|\nabla \AA\left(e_{1}, e_{2}\right)\right|^{2} \geq\left.(1-\varepsilon)|\nabla| \AA\right|^{2}+\left|\partial_{r}^{\top}\right|^{2} O(\exp (-6 r))+O(\exp (-10 r))
$$

and obtain that

$$
\begin{aligned}
\int_{\Sigma_{t}}|\nabla \AA|^{2} d \mu \geq\left.(2-\varepsilon) \int_{\Sigma_{t}}|\nabla| \AA\right|^{2} d \mu & \\
& +\int_{\Sigma_{t}}\left|\partial_{r}^{\top}\right|^{2} O(\exp (-6 r)) d \mu+\int_{\Sigma_{t}} O(\exp (-10 r)) d \mu .
\end{aligned}
$$

To handle the last term we remark that

$$
\int_{\Sigma_{t}}(u-\bar{u})^{2} O(\exp (-3 r)) d \mu \leq o(1) f_{\Sigma_{t}}|\stackrel{\AA}{\mid}|^{2} d \mu,
$$

and hence, we can rewrite equation (8) as

$$
\begin{aligned}
& (1-\varepsilon) \int_{\Sigma_{t}}|\stackrel{\circ}{4}|^{4} d \mu \leq 1 /(2-\varepsilon) \int_{\Sigma_{t}}|\nabla \AA|^{2} d \mu+o(1) f_{\Sigma_{t}}|\AA|^{2} d \mu \\
& +\int_{\Sigma_{t}}\left|\partial_{r}^{\top}\right|^{2} O(\exp (-6 r)) d \mu+\int_{\Sigma_{t}} O(\exp (-10 r)) d \mu .
\end{aligned}
$$

Multiplying this inequality by $(1+\varepsilon) /(1-\varepsilon)$ (with $\varepsilon$ small) and adding to equation (7) we obtain

$$
\begin{aligned}
& f_{\Sigma_{t}}|\stackrel{\AA}{A}|^{2} d \mu+\int_{\Sigma_{t}}|\stackrel{\AA}{A}|^{4} d \mu+\int_{\Sigma_{t}}|\nabla \stackrel{\AA}{A}|^{2} d \mu \leq \int_{\Sigma_{t}}\left|\partial_{r}^{\top}\right|^{2} O(\exp (-4 r)) d \mu \\
& +\int_{\Sigma_{t}} O(\exp (-7 r)) d \mu .
\end{aligned}
$$




\section{$5 \quad$ Intrinsic Geometry}

We study the intrinsic geometry of $\left(\Sigma_{t}\right)_{t \geq 0}$, the foliation of stable spheres with constant mean curvature satisfying condition (1). More precisely, we show

Theorem 5.1. After pulling back by a suitable diffeomorphism from $\Sigma_{t}$ to $S^{2}$, the metric

$$
\hat{g}_{t} \equiv 4 \pi\left|\Sigma_{t}\right|^{-1} g_{t}
$$

can be written as

$$
\exp \left(2 \beta_{t}\right) g_{0}
$$

with

$$
\sup \left|\beta_{t}\right|=O\left(\exp \left(2 \bar{r}_{t}-3 \underline{r}_{t}\right)\right) \quad \text { and } \quad \int_{S^{2}}\left|\nabla \beta_{t}\right|^{2} d \mu_{0}=O\left(\exp \left(4 \bar{r}_{t}-6 \underline{r}_{t}\right)\right)
$$

where the norms are computed with respect to $g_{0}$, the standard round metric on $S^{2}$.

We need to show that the Gaussian curvature $\widehat{K}_{t}$ of $\Sigma_{t}$ (computed with respect to $\left.\hat{g}_{t}\right)$ converges to one with order $O\left(\exp \left(2 \bar{r}_{t}-3 \underline{r}_{t}\right)\right)$. In order to do so, we know

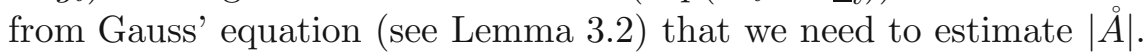

LEMMA 5.2. $|\AA|^{2}$ is uniformly bounded.

Proof. Suppose there is a sequence $t_{i}$ tending to infinity and a sequence of points $x_{i}$ in each $\Sigma_{t_{i}}$ (denoted simply by $\Sigma_{i}$ ) such that

$$
\sup _{\Sigma_{i}}|\AA|=|\AA|\left(x_{i}\right) \equiv 1 / \sigma_{i} \text { and } \lim _{i \rightarrow \infty}|\AA|\left(x_{i}\right)=\infty \text {. }
$$

Consider the sequence of ambient metrics $g_{i}=\sigma_{i}^{-2} g$ and denote the various geometric quantities with respect to $g_{i}$ using an index $i$. Because $H_{i}=\sigma_{i} H$ converges to zero (see Lemma 4.1), we have that for all $i$ sufficiently large

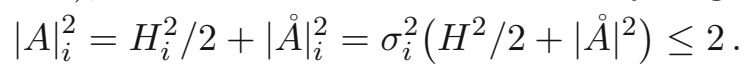

Thus, there exists a universal constant $r_{0}$ for which $B_{r_{0}}^{i}\left(x_{i}\right) \cap \Sigma_{i}$ is the graph over $T_{x_{i}} \Sigma_{i}$ of a function with gradient bounded by one (see, for instance, [CoM])). As a result, there is a uniform constant $C$ such that, for all $s \leq r_{0}$,

$$
C^{-1} s^{2} \leq \int_{B_{s}^{i}\left(x_{i}\right) \cap \Sigma_{i}} d \mu_{i} \leq C s^{2} .
$$

Furthermore, the generalization of Michael-Simon Sobolev inequality proven in $[\mathrm{H}]$ states the existence of some other universal constant $C$ such that, for every compactly supported function $u$,

$$
\left(\int_{\Sigma_{i}} u^{2} d \mu_{i}\right)^{1 / 2} \leq C\left(\int_{\Sigma_{i}}|\nabla u|_{i} d \mu_{i}+\int_{\Sigma_{i}} H_{i}|u| d \mu_{i}\right)
$$

Hence, because $H_{i}$ converges to zero, we have that for all $i$ sufficiently large and every compactly supported function $u$

$$
\left(\int_{B_{r_{0}}^{i}\left(x_{i}\right) \cap \Sigma_{i}} u^{2} d \mu_{i}\right)^{1 / 2} \leq 2 C \int_{B_{r_{0}}^{i}\left(x_{i}\right) \cap \Sigma_{i}}|\nabla u|_{i} d \mu_{i}
$$

Finally, because $|\AA|_{i}$ is uniformly bounded, it follows easily from Lemma 3.3 that

$$
\Delta_{i}|\AA|_{i}^{2} \geq-3|\AA|_{i}^{2}+O(\exp (-3 r)) \text {. }
$$


We now have all the necessary conditions to apply Moser's iteration argument (see, for instance, [L, Lem. 11.1.]) and obtain that, for some constant $C$,

$$
1=|\AA|_{i}^{2}\left(x_{i}\right) \leq C \int_{\Sigma_{i}}|\AA|_{i}^{2} d \mu_{i}+O\left(\exp \left(-3 \underline{r}_{t}\right)\right)=C \int_{\Sigma_{t_{i}}}|\AA|^{2} d \mu+O\left(\exp \left(-3 \underline{r}_{t}\right)\right) .
$$

The last expression converges to zero by Proposition 4.3 and this gives us a contradiction.

Next, we improve the estimate on $|\AA|^{2}$. The idea is to exploit the fact that the hyperbolic metric is conformal to the Euclidean metric on the unit ball. Therefore, each $\Sigma_{t}$ inherits another induced metric. The proof consists in showing that, for this new induced metric, we can apply Moser's iteration argument for $|\AA|^{2}$ and thus bound the supremum by its $L^{2}$ norm (computed with respect to the Euclidean metric).

Proposition 5.3. The following estimate holds

$$
\sup _{\Sigma_{t}}|\stackrel{\AA}{A}|^{2} \leq O\left(\exp \left(2 \bar{r}_{t}-2 \underline{r}_{t}\right)\right) \int_{\Sigma_{t}}\left|\partial_{r}^{\top}\right|^{2} O(\exp (-4 r)) d \mu+O\left(\exp \left(2 \bar{r}_{t}-7 \underline{r}_{t}\right)\right) .
$$

In particular,

$$
\sup _{\Sigma_{t}}|\AA|^{2} \leq O\left(\exp \left(2 \bar{r}_{t}-6 \underline{r}_{t}\right)\right) \text {. }
$$

Proof. Recall that, with respect to the the unit model for the hyperbolic metric, the metric $g$ can be written as

$$
g=\psi^{2}\left(d x^{2}+d y^{2}+d z^{2}\right)+O\left(\psi^{-3}\right)
$$

where

$$
\psi(x)=\frac{2}{1-|x|^{2}} \quad \text { and } \quad \sinh r=\frac{2|x|}{1-|x|^{2}} .
$$

The surfaces $\Sigma_{t}$ converge pointwise to the sphere of radius one and, according to Proposition 4.2 (i)

$$
\lim _{t \rightarrow \infty} \int_{\Sigma_{t}} \exp (-2 r) d \mu=\lim _{t \rightarrow \infty} \int_{\Sigma_{t}} d \sigma=\pi
$$

where $d \sigma$ denotes the surface measure induced by the Euclidean metric. The tracefree part of the second fundamental form and the mean curvature with respect to

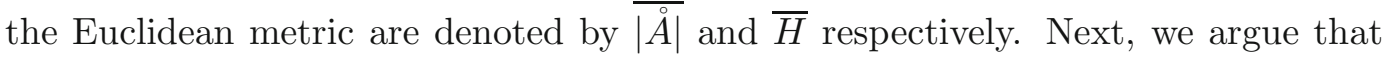
we have the necessary conditions to apply Moser's iteration on $\Sigma_{t}$ with respect to the Euclidean metric.

We start with the remark that

$$
\lim _{t \rightarrow \infty} \int_{\Sigma_{t}} \overline{\mid \AA ्}^{2} d \sigma=0
$$

The reason is that, by conformal invariance of

$$
\int_{\Sigma_{t}}|\AA|^{2} d \mu
$$

the above identity is true if we substitute the Euclidean metric by $\delta \equiv \psi^{-2} g$. Nevertheless, this metric is a lower-order perturbation of the Euclidean metric and so, 
using the formulas derived in [HuI, §7] (more precisely identity (7.10)) the remark follows. Therefore, the Gauss-Bonnet theorem implies that

$$
\lim _{t \rightarrow \infty} \int_{\Sigma_{t}} \bar{H}^{2} d \sigma=16 \pi
$$

We use this to argue that the mean curvature has no concentration points.

LEMma 5.4. For every $\varepsilon_{0}>0$ there is a positive $r_{0}$ such that, for all $t$ sufficiently large and all $x$ in $\Sigma_{t}$,

$$
\int_{\Sigma_{t} \cap \bar{B}_{r_{0}}(x)} \bar{H}^{2} d \sigma \leq \varepsilon_{0} .
$$

Proof. Denote by $\mathcal{H}^{2}$ the 2-dimensional Hausdorff measure and consider the sequence of Radon measures given by

$$
\eta_{i}(A) \equiv \int_{\Sigma_{t} \cap A} \bar{H}^{2} d \sigma
$$

where $A$ is any $\mathcal{H}^{2}$-measurable set. From Allard's compactness theorem, we can extract a sequence $\Sigma_{i}$ that converges to the unit sphere in the varifold sense and, moreover, we can also assume that $\eta_{i}$ converges to a Radon measure $\bar{\eta}$ supported on the unit sphere. Lower semicontinuity implies that, for every $\mathcal{H}^{2}$-measurable set $A$,

$$
\bar{\eta}(A) \geq 4 \mathcal{H}^{2}(A \cap\{|x|=1\}) .
$$

On the other hand,

$$
4 \mathcal{H}^{2}(\{|x|=1\})=16 \pi=\lim _{i \rightarrow \infty} \int_{\Sigma_{i}} \bar{H}^{2} d \sigma=\bar{\eta}(\{|x|=1\}) .
$$

Therefore, an equality in (9) must hold for every $\mathcal{H}^{2}$-measurable set $A$ and this implies the desired result.

An immediate consequence of this lemma combined with the generalization of Michael-Simon Sobolev inequality proven in $[\mathrm{H}]$ is the existence of universal constants $C$ and $r_{0}$ such that, for every Euclidean ball $\bar{B}_{r_{0}}$ centered at a point in $\{|x|=1\}$ and for all $t$ sufficiently large, we have both that

$$
\left(\int_{\Sigma_{t} \cap \bar{B}_{r_{0}}} u^{2} d \sigma\right)^{1 / 2} \leq C \int_{\Sigma_{t} \cap \bar{B}_{r_{0}}}|\bar{\nabla} u| d \sigma
$$

for every compactly supported function $u$, and that

$$
\sqrt{\mathcal{H}^{2}(A)} \leq K \mathcal{H}^{1}(\partial A)
$$

for every open subset $A$ of $\Sigma_{t} \cap \bar{B}_{r_{0}}$ with rectifiable boundary. A standard argument implies the existence of a universal constant $C$ for which

$$
C^{-1} s^{2} \leq \mathcal{H}^{2}\left(\widehat{B}_{s}(x)\right) \leq C s^{2} \text { for all } s \leq r_{0},
$$

where $x$ is in $\Sigma_{t}$ and $\widehat{B}_{s}(x)$ denotes the intrinsic ball of radius $s$.

With respect to the Euclidean Laplacian, the equation for $|\AA|^{2}$ becomes (see Lemma 3.3)

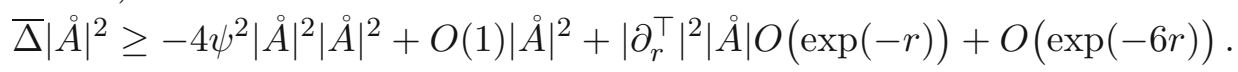

The zeroth order terms are bounded and so, in order to apply Moser's iteration argument, we need to check that $\psi^{2}|\AA|^{2}$ is in $L^{p}$ for some $p>1$. Because $|\AA|$ is 
bounded, we have from Proposition 4.3

$$
\begin{aligned}
\int_{\Sigma_{t}} \psi^{2+\varepsilon}|\AA|^{2+\varepsilon} d \sigma & =\int_{\Sigma_{t}} \psi^{\varepsilon}|\AA|^{2+\varepsilon} d \mu+\int_{\Sigma_{t}}|\AA|^{2+\varepsilon} O(\exp (-(3-\varepsilon) r)) d \mu \\
& =O\left(\exp \left((2+\varepsilon) \bar{r}_{t}-4 \underline{r}_{t}\right)\right),
\end{aligned}
$$

which is bounded for all $\varepsilon$ sufficiently small. Hence, setting

$$
\alpha_{t} \equiv \sup _{\Sigma_{t}}|\AA|
$$

Moser's iteration (see, for instance, [L, Lem.11.1]) implies the existence of some constant $C$ depending on $\varepsilon$ small for which

$$
\alpha_{t}^{2} \leq C \int_{\Sigma_{t}}|\AA|^{2} d \sigma+\alpha_{t}\left(\int_{\Sigma_{t}}\left|\partial_{r}^{\top}\right|^{2+2 \varepsilon} O(\exp (-(1+\varepsilon) r)) d \sigma\right)^{1 /(1+\varepsilon)}+O\left(\exp \left(-6 \underline{r}_{t}\right)\right) \text {. }
$$

Therefore, Proposition 4.2 (iii) implies that

$$
\alpha_{t}^{2} \leq O\left(\exp \left(-2 \underline{r}_{t}\right)\right) \int_{\Sigma_{t}} \mid \stackrel{\AA}{\left.\right|^{2}} d \mu+O\left(\exp \left(-2(3+\varepsilon) /(1+\varepsilon) \underline{r}_{t}\right)\right) .
$$

The result follows from applying Proposition 4.3 and choosing $\varepsilon$ appropriately small.

We can now prove Theorem 5.1.

Proof of Theorem 5.1. Lemma 3.2 and Lemma 4.1 imply that the Gaussian curvature of $\Sigma_{t}$ with respect to $\hat{g}_{t}$ satisfies

$$
\widehat{K}_{t}=1-\left|\Sigma_{t}\right||\stackrel{\AA}{ }|^{2} /(8 \pi)+O\left(\exp \left(2 \underline{r}_{t}-3 \underline{r}_{t}\right)\right) .
$$

As a result, we obtain from Proposition 5.3 that

$$
\widehat{K}_{t}=1+O\left(\exp \left(4 \bar{r}_{t}-6 \underline{r}_{t}\right)\right)+O\left(\exp \left(2 \bar{r}_{t}-3 \underline{r}_{t}\right)\right)=1+O\left(\exp \left(2 \bar{r}_{t}-3 \underline{r}_{t}\right)\right) .
$$

Hence, because we are assuming condition (1), $\widehat{K}_{t}$ converges uniformly to one and this implies that, after pulling back by a diffeomorphism, the metric $\hat{g}_{t}$ can be written as $\exp \left(2 \beta_{t}\right) g_{0}$ where, according to [CY, Lem. 3.7], $\beta_{t}$ converges uniformly to zero and, denoting the coordinate function on $S^{2}$ by $x_{1}, x_{2}$, and $x_{3}$,

$$
\int_{S^{2}} x_{j} \exp \left(2 \beta_{t}\right) d \mu_{0}=0 \quad \text { for } j=1,2,3 \text {. }
$$

Using the smallness of $\beta_{t}$ for $t$ sufficiently large, we have that $\beta_{t}$ satisfies the equation

$$
\begin{aligned}
\Delta_{0} \beta_{t} & =1-\widehat{K}_{t} \exp \left(2 \beta_{t}\right) \\
& =1-\exp \left(2 \beta_{t}\right)+O\left(\exp \left(2 \bar{r}_{t}-3 \underline{r}_{t}\right)\right) \exp \left(2 \beta_{t}\right) \\
& =-2 \beta_{t}+f\left(\beta_{t}\right)+O\left(\exp \left(2 \bar{r}_{t}-3 \underline{r}_{t}\right)\right) \exp \left(2 \beta_{t}\right),
\end{aligned}
$$

where

$$
f(s) \equiv 1+2 s-\exp (2 s)
$$

is such that $\left|f\left(\beta_{t}\right)\right|=O\left(\beta_{t}^{2}\right)$. Therefore,

$$
\begin{aligned}
\bar{\beta}_{t} \equiv f_{S^{2}} \beta_{t} d \mu_{0} & =f_{S^{2}} f\left(\beta_{t}\right) / 2 d \mu_{0}+O\left(\exp \left(2 \bar{r}_{t}-3 \underline{r}_{t}\right)\right) \\
& =O\left(\left|\beta_{t}\right|_{2}^{2}\right)+O\left(\exp \left(2 \bar{r}_{t}-3 \underline{r}_{t}\right)\right)
\end{aligned}
$$


and, combining integration by parts with Cauchy's inequality,

$$
\int_{S^{2}}\left|\nabla \beta_{t}\right|^{2} d \mu_{0} \leq(2+o(1)+1 / 2) \int_{S^{2}} \beta_{t}^{2} d \mu_{0}+O\left(\exp \left(4 \bar{r}_{t}-6 \underline{r}_{t}\right)\right) .
$$

On the other hand, we know that the $L^{2}$ norm of the projection of $\beta_{t}$ on the kernel of $\Delta_{0}+2$ has order $O\left(\left|\beta_{t}\right|_{2}^{2}\right)$ because, from (10), we have that for $j=1,2,3$,

$$
2 \int_{S^{2}} x_{j} \beta_{t} d \mu_{0}=\int_{S^{2}} x_{j} f\left(\beta_{t}\right) d \mu_{0}=O\left(\left|\beta_{t}\right|_{2}^{2}\right) .
$$

Hence, because $\beta_{t}$ converges uniformly to zero,

$$
\int_{S^{2}}\left|\nabla \beta_{t}\right|^{2} d \mu_{0} \geq(6+o(1)) \int_{S^{2}}\left(\beta-\bar{\beta}_{t}\right)^{2} d \mu_{0}
$$

Combining this with (11) and (12) we obtain

$$
\int_{S^{2}} \beta_{t}^{2} d \mu_{0}=O\left(\exp \left(4 \bar{r}_{t}-6 \underline{r}_{t}\right)\right)
$$

and so, by (12), the estimate on the $L^{2}$-norm of the gradient follows. Finally, a simple computation shows that for some constant $C$

$$
\Delta_{0} \beta_{t}^{2} \geq-C \beta_{t}^{2}-O\left(\exp \left(4 \bar{r}_{t}-6 \underline{r}_{t}\right)\right)
$$

Thus, from Moser's iteration, we obtain that for another constant $C$

$$
\sup _{\Sigma_{t}} \beta_{t}^{2} \leq C \int_{S^{2}} \beta_{t}^{2} d \mu_{0}+O\left(\exp \left(4 \bar{r}_{t}-6 \underline{r}_{t}\right)\right)=O\left(\exp \left(4 \bar{r}_{t}-6 \underline{r}_{t}\right)\right) \text {. }
$$

\section{Unique Approximation to Coordinate Spheres}

The purpose of this section is to show that a foliation by stable spheres $\left(\Sigma_{t}\right)_{t \geq 0}$ with constant mean curvature must approach the coordinate spheres $\{|x|=r\}$ when $t$ tends to infinity. Such a result is obviously false in hyperbolic space because we can always apply an isometry to our foliation that changes its center. Hence, we need to use the fact that the ambient manifold is an asymptotically hyperbolic space with nonzero mass. The connection between nonzero mass and uniqueness of the limit for any foliation will be made through the Kazdan-Warner identity. More precisely, we show

Theorem 6.1. Let $\left(\Sigma_{t}\right)_{t \geq 0}$ be a foliation by stable spheres with constant mean curvature satisfying condition (1). Then

Proof. Let

$$
\lim _{t \rightarrow \infty}\left(\bar{r}_{t}-\underline{r}_{t}\right)=0 \quad \text { and } \quad \lim _{t \rightarrow \infty} \int_{\Sigma_{t}}\left|\partial_{r}^{\top}\right|^{2} d \mu=0 .
$$

We want to show that

$$
w_{t}(x) \equiv r(x)-\hat{r}_{t}, \quad \text { where }\left|\Sigma_{t}\right|=4 \pi \sinh ^{2} \hat{r}_{t} .
$$

$$
\lim _{t \rightarrow \infty} w_{t}=0 \quad \text { and } \quad \lim _{t \rightarrow \infty} \int_{\Sigma_{t}}\left|\nabla w_{t}\right|^{2} d \mu=0
$$

An immediate consequence of Proposition 4.2 and Theorem 5.1 is that

$$
\begin{aligned}
\lim _{t \rightarrow \infty} \int_{S^{2}} \exp \left(-2 w_{t}\right) d \mu_{0} & =\lim _{t \rightarrow \infty} \int_{\Sigma_{t}} 4 \pi \exp \left(-2 w_{t}\right) /\left|\Sigma_{t}\right| d \mu \\
& =\lim _{t \rightarrow \infty} \int_{\Sigma_{t}} 4 \exp (-2 r) d \mu=4 \pi
\end{aligned}
$$


We start by deriving a sequence of preliminary results. Combining the first identity in Proposition 3.4 with both Lemma 4.1 and Theorem 5.1, we obtain that the Laplacian of $w_{t}$ with respect to the standard round metric $g_{0}$ is given by

$$
\Delta_{0} w_{t}=\exp \left(-2 w_{t}\right)-1+P_{t},
$$

where

$$
\begin{aligned}
P_{t}=-2\left|\nabla_{0} w_{t}\right|^{2} \exp (-2 r)+(4 \pi)^{-1}\left|\Sigma_{t}\right| \exp \left(2 \beta_{t}\right)\left(1-\left\langle\partial_{r}, \nu\right\rangle\right)^{2} & \\
& +\exp \left(2 \beta_{t}\right)\left(1-\left\langle\partial_{r}, \nu\right\rangle\right)+\left(\exp \left(-2 w_{t}\right)+1\right) O\left(\exp \left(2 \bar{r}_{t}-3 \underline{r}_{t}\right)\right) \\
& +O\left(\exp \left(-2 \underline{r}_{t}\right)\right) .
\end{aligned}
$$

Furthermore, Proposition 4.2 and (13) imply

LEMMA 6.2.

$$
\int_{\Sigma_{t}}|P| d \mu_{0}=O\left(\exp \left(-\underline{r}_{t}\right)\right)+O\left(\exp \left(2 \bar{r}_{t}-3 \underline{r}_{t}\right)\right) .
$$

Note that if $\hat{g}_{t}$ was the round metric and $P_{t} \equiv 0$, then solutions to equation (14) would correspond to constant scalar curvature metrics. Because there is no compactness for the set of such solutions, we cannot expect to derive apriori estimates solely from equation (14). In order to have good estimates we need to prevent $\exp \left(-2 w_{t}\right)$ from concentrating at any point. This will be achieved because of the lemma we present next. It uses the Kazdan-Warner identity $[\mathrm{K}]$ combined with the fact that the mass of the ambient manifold is not zero and that $\Sigma_{t}$ has constant mean curvature.

LEMma 6.3. For each of the standard coordinate functions $x_{1}, x_{2}, x_{3}$ on $S^{2}$ we have

$$
\begin{aligned}
m \int_{S^{2}} x_{i} \exp \left(-3 w_{t}\right) d \mu_{0}=O\left(\operatorname { e x p } \left(2 \bar{r}_{t}\right.\right. & \left.\left.-3 \underline{r}_{t}\right)\right) \int_{S^{2}} x_{i} \exp \left(-3 w_{t}\right) d \mu_{0} \\
& +O\left(\exp \left(3 \bar{r}_{t}-4 \underline{r}_{t}\right)\right)+O\left(\exp \left(5 \bar{r}_{t}-6 \underline{r}_{t}\right)\right) .
\end{aligned}
$$

In particular, for $i=1,2,3$,

$$
\lim _{t \rightarrow \infty} \int_{S^{2}} x_{i} \exp \left(-3 w_{t}\right) d \mu_{0}\left(\int_{S^{2}} \exp \left(-3 w_{t}\right) d \mu_{0}\right)^{-1}=0 .
$$

Proof. Recall that the Gaussian curvature of $\Sigma_{t}$ with respect to $\hat{g}_{t}$ is denoted by $\widehat{K}_{t}$. Kazdan-Warner identity $[\mathrm{K}]$ says that, for the standard coordinate functions $x_{1}, x_{2}, x_{3}$ on $S^{2}$, the following identity holds for each $i=1,2,3$,

$$
\int_{S^{2}}\left\langle\nabla \widehat{K}_{t}, \nabla x_{i}\right\rangle \exp \left(2 \beta_{t}\right) d \mu_{0}=0
$$

which, since $\Delta x_{i}=2 x_{i}$, is equivalent to

$$
\int_{S^{2}} x_{i} \widehat{K}_{t} \exp \left(2 \beta_{t}\right) d \mu_{0}-\int_{S^{2}} \widehat{K}_{t}\left\langle\nabla \beta_{t}, \nabla x_{i}\right\rangle \exp \left(2 \beta_{t}\right) d \mu_{0}=0 .
$$

We saw in section 5 that

$$
\widehat{K}_{t}=1+O\left(\exp \left(2 \bar{r}_{t}-3 \underline{r}_{t}\right)\right)
$$

and this implied that (see Theorem 5.1)

$$
\beta_{t}=O\left(\exp \left(2 \bar{r}_{t}-3 \underline{r}_{t}\right)\right) \quad \text { and } \quad \int_{S^{2}}\left|\nabla \beta_{t}\right|^{2} d \mu_{0}=O\left(\exp \left(4 \bar{r}_{t}-6 \underline{r}_{t}\right)\right) \text {. }
$$


Thus,

and so

$$
\int_{S^{2}}\left|\left\langle\nabla \beta_{t}, \nabla x_{i}\right\rangle\right| d \mu_{0} \leq O\left(\exp \left(2 \bar{r}_{t}-3 \underline{r}_{t}\right)\right)
$$

$$
\begin{aligned}
\int_{S^{2}} \widehat{K}_{t}\left\langle\nabla \beta_{t}, \nabla x_{i}\right\rangle \exp \left(2 \beta_{t}\right) d \mu_{0}=\int_{S^{2}}\left\langle\nabla \beta_{t}, \nabla x_{i}\right\rangle d \mu_{0}+O\left(\exp \left(4 \bar{r}_{t}-6 \underline{r}_{t}\right)\right) \\
=\int_{S^{2}} 2 \beta_{t} x_{i} d \mu_{0}+O\left(\exp \left(4 \bar{r}_{t}-6 \underline{r}_{t}\right)\right)=O\left(\exp \left(4 \bar{r}_{t}-6 \underline{r}_{t}\right)\right),
\end{aligned}
$$

where the last equality comes from the fact that (recall proof of Theorem 5.1)

$$
\int_{S^{2}} x_{j} \exp \left(2 \beta_{t}\right) d \mu_{0}=0 \text { for } j=1,2,3 .
$$

Therefore, the Kazdan-Warner identity becomes

$$
\int_{S^{2}} x_{i} \widehat{K}_{t} \exp \left(2 \beta_{t}\right) d \mu_{0}=O\left(\exp \left(4 \bar{r}_{t}-6 \underline{r}_{t}\right)\right) \quad \text { for } i=1,2,3 \text {. }
$$

From Lemma 3.2 we know that

$$
\begin{array}{r}
4 \pi \widehat{K}_{t}=\left|\Sigma_{t}\right|\left(H^{2}-4\right) / 4+m\left|\Sigma_{t}\right| / \sinh ^{3} r-3 m\left|\partial_{r}^{\top}\right|^{2}\left|\Sigma_{t}\right| /\left(2 \sinh ^{3} r\right) \\
-|\AA|^{2}\left|\Sigma_{t}\right| / 2+O\left(\exp \left(2 \bar{r}_{t}-5 r\right)\right),
\end{array}
$$

and hence, because $\Sigma_{t}$ has constant mean curvature, we obtain from (16) and (15) that

$$
\begin{aligned}
& m \int_{S^{2}} x_{i}\left|\Sigma_{t}\right|^{3 / 2} \sinh ^{-3} r \exp \left(2 \beta_{t}\right) d \mu_{0} \\
&=\int_{\Sigma_{t}} 3 / 2 m\left|\partial_{r}^{\top}\right|^{2}\left|\Sigma_{t}\right|^{3 / 2} \sinh ^{-3} r \exp \left(2 \beta_{t}\right) d \mu_{0} \\
& \quad+\int_{S^{2}}\left|\Sigma_{t}\right|^{3 / 2}|\AA|^{2} / 2 \exp \left(2 \beta_{t}\right) d \mu_{0} \\
&+O\left(\exp \left(3 \bar{r}_{t}-5 \underline{r}_{t}\right)\right)+O\left(\exp \left(5 \bar{r}_{t}-6 \underline{r}_{t}\right)\right) .
\end{aligned}
$$

The first assertion in the result follows because, due to Theorem 5.1, Proposition 4.2, and Proposition 4.3,

$$
\begin{gathered}
\int_{\Sigma_{t}} 3 m\left|\partial_{r}^{\top}\right|^{2}\left|\Sigma_{t}\right|^{3 / 2} /\left(2 \sinh ^{3} r\right) d \mu_{0}=O\left(\exp \left(\bar{r}_{t}-3 \underline{r}_{t}\right)\right), \\
\int_{S^{2}}|\stackrel{\AA}{ }|^{2}\left|\Sigma_{t}\right|^{3 / 2} d \mu_{0}=O\left(\exp \left(3 \bar{r}_{t}-4 \underline{r}_{t}\right)\right)
\end{gathered}
$$

and

$$
\begin{array}{r}
m \int_{S^{2}} x_{i}\left|\Sigma_{t}\right|^{3 / 2} / \sinh ^{3} r d \mu_{0}=(4 \pi)^{3 / 2} m \int_{S^{2}} x_{i} \exp \left(-3 w_{t}\right) d \mu_{0} \\
\quad+O\left(\bar{r}_{t}-3 \underline{r}_{t}\right)+O\left(-4 \underline{r}_{t}\right) .
\end{array}
$$

The second assertion of the lemma follows from Hölder's inequality and condition (1).

We use this lemma to prove

Proposition 6.4. The functions $w_{t}$ are uniformly bounded in $W^{1,2}$. 
Proof. Given a smooth function $u$ on $S^{2}$, denote

$$
S[u] \equiv \int_{S^{2}}\left|\nabla_{0} u\right|^{2} d \mu_{0}-2 \int_{S^{2}} u d \mu_{0} .
$$

This functional has the property that, given $F$ a conformal transformation of $S^{2}$, then

$$
S[u]=S[v] \quad \text { where } F^{*}\left(\exp (-2 u) g_{0}\right)=\exp (-2 v) g_{0} .
$$

This invariance can be used in the same way as in [CY, Prop.4.1] in order to show LEMma 6.5. $S\left[w_{t}\right]$ is bounded independently of $t$.

The proof will be given in the Appendix. From integration by parts in (14) we obtain

$$
\int_{S^{2}}\left|\nabla_{0} w_{t}\right|^{2} d \mu_{0}-\int_{S^{2}} w_{t} d \mu_{0}=-\int_{S^{2}} w_{t} \exp \left(-2 w_{t}\right) d \mu_{0}-\int_{S^{2}} w_{t} P_{t} d \mu_{0} .
$$

The last term on the right-hand side converges to zero because Lemma 6.2 implies that

Set

$$
\int_{S^{2}}\left|w_{t} P_{t}\right| d \mu_{0} \leq\left|\bar{r}_{t}-\underline{r}_{t}\right|\left(O\left(\exp \left(-\underline{r}_{t}\right)\right)+O\left(\exp \left(2 \bar{r}_{t}-3 \underline{r}_{t}\right)\right)\right) .
$$

$$
V_{t} \equiv \int_{S^{2}} \exp \left(-2 w_{t}\right) d \mu_{0},
$$

which we know converges to $4 \pi$. Using Jensen's inequality we obtain that, for all $t$ sufficiently large,

$$
\int_{S^{2}}\left|\nabla_{0} w_{t}\right|^{2} d \mu_{0}-\int_{S^{2}} w_{t} d \mu_{0} \leq V_{t} \log \left(V_{t}^{-1} \int_{S^{2}} \exp \left(-3 w_{t}\right) d \mu_{0}\right)+1 .
$$

We estimate the first term on the right-hand side using a slightly modified form of Aubin's inequality [A, Th.6].

Lemma 6.6 (Aubin). Given a smooth function $u$ in $S^{2}$ such that, for all $i=1,2,3$,

$$
\left|\int_{S^{2}} x_{i} \exp (-3 u) d \mu_{0}\right|\left(\int_{S^{2}} \exp (-3 u) d \mu_{0}\right)^{-1} \leq 1 / 2,
$$

then, for every $\delta>0$ there is $C(\delta)$ so that

$$
4 \pi \log f_{S^{2}} \exp (-3 u) d \mu_{0} \leq\left(\frac{9}{8}+\delta\right) \int_{S^{2}}\left|\nabla_{0} u\right|^{2} d \mu_{0}-3 \int_{S^{2}} u d \mu_{0}+C(\delta) .
$$

Aubin's proof extends to this setting with obvious modifications. Lemma 6.3 implies that we can apply this result and so, combining it with (18), we have

$$
C(\delta) \leq\left((9 / 8+\delta) V_{t} /(4 \pi)-1\right) \int_{S^{2}}\left|\nabla_{0} w_{t}\right|^{2} d \mu_{0}-\left(3 V_{t} /(4 \pi)-1\right) \int_{S^{2}} w_{t} d \mu_{0}
$$

for some constant $C(\delta)$. Therefore, using Lemma 6.5, we obtain the existence of some constant $C(\delta)$ for which

$$
C(\delta) \leq\left((2 \delta-3 / 4) V_{t} /(4 \pi)-1\right) \int_{S^{2}} w_{t} d \mu_{0},
$$

and thus, choosing $\delta$ sufficiently small, we get that

$$
\int_{S^{2}} w_{t} d \mu_{0}
$$


is uniformly bounded from above. We already know it is trivially bounded from below because $V_{t}$ converges to $4 \pi$ and so, we can apply Lemma 6.5 and conclude that

$$
\int_{S^{2}}\left|\nabla_{0} w_{t}\right|^{2} d \mu_{0}
$$

is uniformly bounded above. The result follows from Poincaré inequality.

For any sequence $t_{j}$ tending to infinity, we can extract a subsequence so that $w_{t_{j}}$ converges weakly in $W^{1,2}$ to $w_{0}$. Furthermore, we know that from Trudinger's inequality [T] (see also [C2, Cor. 1.8]), $\exp \left(-p w_{t_{j}}\right)$ converges in $L^{1}$ to $\exp \left(-p w_{0}\right)$ for any $p$. Therefore, for $i=1,2,3$,

$$
m \int_{S^{2}} x_{i} \exp \left(-3 w_{0}\right) d \mu_{0}=0 \text { and } \int_{S^{2}} \exp \left(-2 w_{0}\right) d \mu_{0}=1 .
$$

On the other hand, it is easy to recognize from (14) that $w_{0}$ satisfies weakly

$$
\Delta_{0} w_{0}=\exp \left(-2 w_{0}\right)-1 \text {, }
$$

and so, the identities in (19) imply that $w_{0}=0$, i.e. $w_{t}$ converges weakly in $W^{1,2}$ to zero. Hence, from Rellich's theorem and integration by parts formula (17) we obtain

$$
\lim _{t \rightarrow \infty} \int_{S^{2}}\left|\nabla_{0} w_{t}\right|^{2} d \mu_{0}=\lim _{t \rightarrow \infty} \int_{S^{2}} w_{t}^{2} d \mu_{0}=0 .
$$

We argue next that $w_{t}$ converges to zero uniformly. We start with LEMMA 6.7.

$$
\lim _{t \rightarrow \infty} \sup _{\Sigma_{t}}\left|\partial_{r}^{\top}\right|^{2}=0
$$

Proof. Note that we have from Proposition 4.2

$$
\lim _{t \rightarrow \infty} \int_{\Sigma_{t}}\left|\partial_{r}^{\top}\right|^{2} d \mu=0 .
$$

Arguing as in the proof of Proposition 3.4 and using the fact that the norm of the second fundamental $\mathrm{rm}$ of $\Sigma_{i}$ is bounded, it is straightforward to see that

$$
\left|\nabla\left\langle\nu, \partial_{r}\right\rangle\right| \leq C\left|\partial_{r}^{\top}\right|+O(\exp (-3 r))
$$

for some constant $C$. Combining Proposition 3.4 with the Bochner formula for $|\nabla r|^{2}$ we obtain that, for some other constant $\mathrm{C}$ and for all $t$ sufficiently large,

$$
\begin{aligned}
\Delta\left|\partial_{r}^{\top}\right|^{2} \geq & -C\left|\partial_{r}^{\top}\right|^{2}+2|\nabla \nabla r|^{2}-|\nabla \nabla r|\left|\partial_{r}^{\top}\right|^{2} O(\exp (-2 r)) \\
& +\left|\partial_{r}^{\top}\right| O(\exp (-3 r)) \\
\geq & -C\left|\partial_{r}^{\top}\right|^{2}+O(\exp (-6 r)) .
\end{aligned}
$$

Because the second fundamental form of $\Sigma_{t}$ is bounded, we can invoke the same reasons as in Lemma 5.2 in order find $r_{0}$ so that, for all $t$ sufficiently large and all $x$ in $\Sigma_{t}$, we can apply Moser's iteration argument in $B_{r_{0}}(x) \cap \Sigma_{t}$ and conclude that

$$
\sup _{\Sigma_{t}}\left|\partial_{r}^{\top}\right|^{2} \leq C \int_{\Sigma_{t}}\left|\partial_{r}^{\top}\right|^{2} d \mu+O(\exp (-6 r))
$$

where $C$ is a constant independent of $t$.

An immediate consequence of this lemma, Proposition 4.2, and the identity $\left|\partial_{r}^{\top}\right|^{2}=1-\left\langle\nu, \partial_{r}\right\rangle^{2}+O(\exp (-5 r))$ is 
LEMMA 6.8. For all $t$ sufficiently large

$$
O(\exp (-5 r))+\left|\partial_{r}^{\top}\right|^{2} / 3 \leq 1-\left\langle\nu, \partial_{r}\right\rangle \leq\left|\partial_{r}^{\top}\right|^{2} / 2+O(\exp (-5 r)) .
$$

A simple computation using identity (14) shows that

$$
\Delta_{0} w_{t}^{2} \geq 8 \pi w_{t}\left(\exp \left(-2 w_{t}\right)-1\right)+2 P_{t} w_{t}
$$

and we want to write this inequality as

$$
\Delta_{0} w_{t}^{2} \geq-C w_{t}^{2}-\left(\text { finite sum of positive terms } f_{i}\right),
$$

where $C$ is a $t$-independent constant and each term $f_{i}$ has its $L^{p}$ norm converging to zero for some $p>1$. We can then apply Moser's iteration argument and conclude that, for some constant $C$,

$$
\sup w_{t}^{2} \leq C \int_{S^{2}} w_{t}^{2} d \mu_{0}+C \sum_{i}\left|f_{i}\right|_{L^{p}}
$$

This implies uniform convergence of $w_{t}$ to zero.

In what follows, $\mathrm{C}$ is a generic constant independent of $t$. The first term in (20) can be easily estimated as

$$
w_{t}\left(\exp \left(-2 w_{t}\right)-1\right) \geq-w_{t}^{2} / 2-\left(\exp \left(-2 w_{t}\right)-1\right)^{2} / 2,
$$

where the term with the exponential converges to zero in any $L^{p}$-norm due to Trudinger's inequality [T]. Looking at the expression of $P_{t} w_{t}$ (see (14)), we have that the first term and the third term cause no problem because

$$
\begin{aligned}
-\left|\nabla_{0} w_{t}\right|^{2} \exp (-2 r) w_{t} & =O(1)\left|\partial_{r}^{\top}\right|^{2} \exp \left(-2 w_{t}\right) w_{t}+w_{t} O\left(\exp \left(-2 \underline{r}_{t}\right)\right) \\
& \geq-C w_{t}^{2}-C\left|\partial_{r}^{\top}\right|^{4} \exp \left(-4 w_{t}\right)+O\left(\exp \left(-4 \underline{r}_{t}\right)\right),
\end{aligned}
$$

and also

$$
\left(1-\left\langle\partial_{r}, \nu\right\rangle\right) w_{t} \geq-w_{t}^{2} / 2-\left(1-\left\langle\partial_{r}, \nu\right\rangle\right)^{2} / 2 \geq-w_{t}^{2} / 2-\left|\partial_{r}^{\top}\right|^{4} / 8
$$

The same sort of estimate works for the last two terms, and to handle the second term we note that, by Proposition 4.2,

$$
\int_{S^{2}}\left|\nabla_{0} w\right|^{2}\left|\partial_{r}^{\top}\right|^{2} d \mu_{0} \leq 9 \int_{\Sigma_{t}}\left(1-\left\langle\partial_{r}, \nu\right\rangle+O(\exp (-5 r))\right)^{2} d \mu=O\left(\exp \left(-\underline{r}_{t}\right)\right) .
$$

Hence, for some constant C,

$$
\begin{aligned}
\int_{S^{2}}\left(\left|\Sigma_{t}\right|\left(1-\left\langle\partial_{r}, \nu\right\rangle\right)^{2}\left|w_{t}\right|\right)^{1+\varepsilon} d \mu_{0} & \leq C \int_{S^{2}}\left|\nabla_{0} w\right|^{2+2 \varepsilon}\left|\partial_{r}^{\top}\right|^{2+2 \varepsilon}\left|w_{t}\right|^{1+\varepsilon} d \mu_{0} \\
& \leq C \int_{S^{2}}\left|\nabla_{0} w\right|^{2+2 \varepsilon}\left|\partial_{r}^{\top}\right|^{2-2 \varepsilon}\left|w_{t}\right|^{1+\varepsilon} d \mu_{0} \\
& =C\left|\Sigma_{t}\right|^{\varepsilon} \int_{S^{2}}\left|\nabla_{0} w\right|^{2}\left|\partial_{r}^{\top}\right|^{2}\left|w_{t}\right|^{1+\varepsilon} d \mu_{0} \\
& \leq\left|\bar{r}_{t}-\underline{r}_{t}\right|^{1+\varepsilon} O\left(\exp \left(2 \varepsilon \bar{r}_{t}-\underline{r}_{t}\right)\right)
\end{aligned}
$$

and, because we are assuming condition (1), we obtain that, for some sufficiently small $\varepsilon$,

$$
\lim _{t \rightarrow \infty} \int_{S^{2}}\left(\left|\Sigma_{t}\right|\left(1-\left\langle\partial_{r}, \nu\right\rangle\right)^{2}\left|w_{t}\right|\right)^{1+\varepsilon} d \mu_{0}=0 .
$$




\section{Strong Approximation to Coordinate Spheres}

We will start by arguing that we can choose $\tilde{r}_{t}$ such that

$$
H=2 \cosh \tilde{r}_{t} / \sinh \tilde{r}_{t}-m / \sinh ^{3} \tilde{r}_{t}+o\left(\exp \left(-4 \tilde{r}_{t}\right)\right) \text {. }
$$

We will then show that, with respect to $\hat{g}_{t}, \Sigma_{t}$ is $o\left(\exp \left(-\underline{r}_{t}\right)\right)$ "close" to $\left\{|x|=\tilde{r}_{t}\right\}$. This is exactly the rate we need in order to prove uniqueness for the constant mean curvature foliation. Being more precise, rename the family of functions $w_{t}$ on $\Sigma_{t}$ by

$$
w_{t}(x) \equiv r(x)-\tilde{r}_{t} .
$$

This section is devoted to show

Theorem 7.1. With respect to the metric $\hat{g}_{t}$,

$$
\left|w_{t}\right|_{C^{2, \alpha}}=o\left(\exp \left(-\underline{r}_{t}\right)\right) .
$$

Before proving Theorem 7.1 we need to argue that $\tilde{r}_{t}$ is well defined. In order to do so, the following proposition is important.

Proposition 7.2. The following identity holds for each $\Sigma_{t}$ :

$$
\sup _{\Sigma_{t}}\left|\partial_{r}^{\top}\right|^{2}=o\left(\exp \left(-2 \underline{r}_{t}\right)\right) \text {. }
$$

Proof. Direct computation implies the following gradient estimate

LEMMA 7.3 .

$$
\left.\left.|\nabla| \partial_{r}^{\top}\right|^{2}\left|\leq\left(4 \exp (-2 r)-4 \pi /\left|\Sigma_{t}\right|\right)\right| \partial_{r}^{\top}|+3| \partial_{r}^{\top}\right|^{3}+\left|\partial_{r}^{\top}\right| o\left(\exp \left(-2 \underline{r}_{t}\right)\right) .
$$

Proof. Because $g$ is a perturbation of the hyperbolic metric, if we decompose a unit tangent vector $V$ as $\bar{V}+\beta \partial_{r}$, where $\bar{V}$ has no $\partial_{r}$ component, then $|\beta| \leq$ $\left|\partial_{r}^{\top}\right|+O\left(\exp \left(-3 \underline{r}_{t}\right)\right)$ and so

$$
\left|\left\langle V-\bar{V}, \partial_{r}^{\top}\right\rangle\right| \leq\left|\partial_{r}^{\top}\right|^{3}+\left|\partial_{r}^{\top}\right|^{2} O\left(\exp \left(-3 \underline{r}_{t}\right)\right) .
$$

Denoting the connection with respect to the hyperbolic metric by $\bar{\nabla}$, we can estimate

$$
\left\langle V, \nabla\left|\partial_{r}^{\top}\right|^{2}\right\rangle=2\left\langle\bar{\nabla}_{V} \partial_{r}, \partial_{r}^{\top}\right\rangle-2\left\langle\partial_{r}, \nu\right\rangle\left\langle\nabla_{V} \nu, \partial_{r}^{\top}\right\rangle+\left|\partial_{r}^{\top}\right| O\left(\exp \left(-3 \underline{r}_{t}\right)\right) .
$$

Due to Theorem 6.1 and Proposition 5.3, we know that

Hence,

$$
\sup _{\Sigma_{t}}|\AA|=o\left(\exp \left(-2 \underline{r}_{t}\right)\right) \text {. }
$$

$$
\begin{aligned}
\left\langle V, \nabla\left|\partial_{r}^{\top}\right|^{2}\right\rangle= & 2(\cosh r / \sinh r)\left\langle\bar{V}, \partial_{r}^{\top}\right\rangle-\left\langle\partial_{r}, \nu\right\rangle\left\langle V, \partial_{r}^{\top}\right\rangle H \\
& \quad+o\left(\exp \left(-2 \underline{r}_{t}\right)\right)\left|\partial_{r}^{\top}\right| \\
\leq & (2 \cosh r / \sinh r-H)\left|\partial_{r}^{\top}\right|+H\left(1-\left\langle\partial_{r}, \nu\right\rangle\right)\left\langle\bar{V}, \partial_{r}^{\top}\right\rangle \\
& \quad+H\left\langle\partial_{r}, \nu\right\rangle\left\langle\bar{V}-V, \partial_{r}^{\top}\right\rangle+o\left(\exp \left(-2 \underline{r}_{t}\right)\right)\left|\partial_{r}^{\top}\right| \\
\leq & \left(4 \exp (-2 r)-4 \pi /\left|\Sigma_{t}\right|\right)\left|\partial_{r}^{\top}\right|+3\left|\partial_{r}^{\top}\right|^{3}+\left|\partial_{r}^{\top}\right| o\left(\exp \left(-2 \underline{r}_{t}\right)\right),
\end{aligned}
$$

where in the last inequality we used Lemma 4.1, Lemma 6.8, and (21).

Choose $p$ in $\Sigma_{t}$ so that $\left|\partial_{r}^{\top}\right|(p)=0$. Given $q$ in $\Sigma_{t}$, denote by $\gamma(s)$ the unit speed geodesic connecting $p$ to $q$ and let $f(s)=\left|\partial_{r}^{\top}\right|(\gamma(s))$. From Lemma 7.3 we have

$$
\begin{aligned}
\left|\frac{d}{d s} f^{2}\right| & \leq\left(|4 \pi /| \Sigma_{t}|-4 \exp (-2 r)|+o\left(\exp \left(-2 \underline{r}_{t}\right)\right)\right) f+3 f^{3} \\
& \leq \alpha_{t}^{2} f+3 f^{3}
\end{aligned}
$$


where

If we set

$$
\alpha_{t}^{2}=\sup _{\Sigma_{t}}|4 \pi /| \Sigma_{t}|-4 \exp (-2 r)|+o\left(\exp \left(-2 \underline{r}_{t}\right)\right)
$$

then

$$
V(s) \equiv \alpha_{t} \tan \left((3 / 4)^{1 / 2} \alpha_{t} s\right) / \sqrt{3}
$$

$$
\left(V^{2}\right)^{\prime}=\alpha_{t}^{2} V+3 V^{2}
$$

and so, while $V$ is well defined, we have $f \leq V$ because $f(p)=0$. Moreover, $\operatorname{diam}\left(\hat{g}_{t}\right)$ converges to 2 and therefore

$$
\lim _{t \rightarrow \infty} \alpha_{t}^{2} \operatorname{diam}^{2}\left(g_{t}\right)=\lim _{t \rightarrow \infty} \alpha_{t}^{2}\left|\Sigma_{t}\right| \pi^{-1} \leq \lim _{t \rightarrow \infty} \sup _{\Sigma_{t}}|4-4| \Sigma_{t}\left|\pi^{-1} \exp \left(-2 \underline{r}_{t}\right)\right|=0,
$$

where the last equality follows from Theorem 6.1. Hence, we obtain for all $t$ sufficiently large

$$
\left|\partial_{r}^{\top}\right|(q)=f(q) \leq V\left(\operatorname{diam}\left(g_{t}\right) / 2\right) \leq \alpha_{t}=o\left(\exp \left(-\underline{r}_{t}\right)\right) .
$$

The decay for $\left|\partial_{r}^{\top}\right|$ proved above combined with Lemma 6.8 allow us to write the second identity in Proposition 3.4 as

$$
\Delta r=2 \cosh r / \sinh r-m / \sinh ^{3} r-H+P,
$$

where $|P|=o(\exp (-4 r))$. Thus, we obtain after integration

$$
H=f_{\Sigma_{t}} 2 \cosh r / \sinh r-m / \sinh ^{3} r d \mu+f_{o}(\exp (-4 r)) d \mu,
$$

and so, the existence of $\tilde{r}_{t}$ satisfying $\underline{r}_{t} \leq \tilde{r}_{t} \leq \bar{r}_{t}$ follows easily.

We also remark that a careful inspection of the term $P$ shows that

$$
|\nabla P|=o(\exp (-5 r))
$$

because, as can be seen from Lemma 6.8, Lemma 7.3, and Proposition 7.2, we have

$$
\left|\nabla\left\langle\partial_{r}, \nu\right\rangle\right|=o(\exp (-3 r)) \text { and }\left.|\nabla| \partial_{r}^{\top}\right|^{2} \mid=o(\exp (-3 r)) \text {. }
$$

Proof of Theorem 7.1. We know from Theorem 6.1 that $w_{t}$ converges to zero uniformly and thus, the expansion for $H$ implies

$$
\begin{aligned}
\Delta r= & 4 \exp \left(-2 \tilde{r}_{t}\right)\left(\exp \left(-2 w_{t}\right)-1\right)-8 m \exp \left(-3 \tilde{r}_{t}\right)\left(\exp \left(-3 w_{t}\right)-1\right) \\
& +o(\exp (-4 r)) \\
=- & 8 w_{t} \exp \left(-2 \tilde{r}_{t}\right)+f\left(w_{t}\right) O(\exp (-2 r))+w_{t} O(\exp (-3 r)) \\
& +o(\exp (-4 r)),
\end{aligned}
$$

where

$$
f(x) \equiv \exp (-2 x)-1+2 x .
$$

Hence, from Theorem 5.1, the above identity translates to

$$
\Delta_{0} w_{t}+\left(2 \exp \left(2 \hat{r}_{t}-2 \tilde{r}_{t}+2 \beta_{t}\right)+o(1)\right) w_{t}=f\left(w_{t}\right) O(1)+o(\exp (-2 r)) .
$$

When $t$ tends to infinity the above operator converges to $\Delta_{0}+2$. Therefore, in order to get a good control of $w_{t}$ we need to control the projection of $w_{t}$ on the kernel of $\Delta_{0}+2$. This is achieved using the following improvement of Lemma 6.3

LEMMA 7.4.

$$
\int_{S^{2}} w_{t} x_{i} d \mu_{0}=o\left(\exp \left(-\underline{r}_{t}\right)\right)+\left|w_{t}\right|_{2}^{2} O(1), \quad i=1,2,3
$$


Proof. We saw in Lemma 7.3 that $|\AA|^{2}$ has order $o\left(\exp \left(-4 \underline{r}_{t}\right)\right)$ and so, we obtain from Lemma 3.2 and Proposition 7.2 that

$$
\widehat{K}_{t}=1+o\left(\exp \left(-\underline{r}_{t}\right)\right) .
$$

This implies that (check proof of Theorem 5.1)

$$
\exp \left(2 \beta_{t}\right)=1+o\left(\exp \left(-\underline{r}_{t}\right)\right)
$$

and thus, an inspection of the proof of Lemma 6.3 shows that

$$
m \int_{S^{2}} \exp \left(-3 w_{t}\right) x_{i} d \mu_{0}=o\left(\exp \left(-\underline{r}_{t}\right)\right), \quad i=1,2,3 .
$$

This implies the desired result.

As a result, if we decompose $w_{t}$ as $u_{t}+v_{t}$, where $u_{t}$ is in the kernel of $\Delta_{0}+2$ and $v_{t}$ is perpendicular to $u_{t}$, we obtain from the previous lemma that

$$
\left|u_{t}\right|_{C^{2, \alpha}}=o\left(\exp \left(-\underline{r}_{t}\right)\right)+\left|w_{t}\right|_{2}^{2} O(1),
$$

and thus, because $u_{t}$ converges to zero uniformly,

$$
\left|u_{t}\right|_{C^{2, \alpha}}=o\left(\exp \left(-\underline{r}_{t}\right)\right)+\left|v_{t}\right|_{2}^{2} O(1) .
$$

On the other hand, we have that $v_{t}$ satisfies

$$
\Delta_{0} v_{t}+(2+o(1)) v_{t}=u_{t} o(1)+f\left(w_{t}\right) O(1)+o(\exp (-2 r)) .
$$

Looking at the way the terms in this equation were derived and using (22) we see that

and

$$
\left|u_{t} o(1)\right|_{C^{0, \alpha}}=o(1)\left|u_{t}\right|_{C^{0, \alpha}}, \quad\left|f\left(w_{t}\right) O(1)\right|_{C^{0, \alpha}} \leq O(1)\left|w_{t}\right|_{C^{0, \alpha}}^{2},
$$

$$
|o(\exp (-2 r))|_{C^{0, \alpha}}=o\left(\exp \left(-2 \underline{r}_{t}\right)\right) .
$$

Hence, from Schauder estimates, we have that for some constant $C$

$$
\left|v_{t}\right|_{C^{2, \alpha}} \leq o(1)\left|u_{t}\right|_{C^{0, \alpha}}+C\left|w_{t}\right|_{C^{0, \alpha}}^{2}+C \sup \left|v_{t}\right|+o\left(\exp \left(-2 \underline{r}_{t}\right)\right)
$$

Moreover, $\left|w_{t}\right|_{C^{0, \alpha}}$ converges to zero (see Proposition 7.2) and hence we have from (23)

$$
\left|v_{t}\right|_{C^{2, \alpha}} \leq \sup \left|v_{t}\right|+o(\exp (-r)) .
$$

Using the same arguments as in the proof of Theorem 5.1, we can see that the orthogonality condition of $v_{t}$, identity (23), and equation (24), imply the estimate

$$
\int_{S^{2}} w_{t}^{2} d \mu_{0}=o\left(\exp \left(-2 \underline{r}_{t}\right)\right) \text {. }
$$

A simple computation shows that, for some constant $C$,

$$
\Delta_{0} v_{t}^{2} \geq-C v_{t}^{2}-C u_{t}^{2}+o\left(\exp \left(-2 \underline{r}_{t}\right)\right)
$$

and thus, using Moser's iteration we obtain

$$
\sup v_{t}^{2} \leq C \int_{S^{2}} w_{t}^{2} d \mu_{0}+C \sup u_{t}^{2}+o\left(\exp \left(-2 \underline{r}_{t}\right)\right)=o\left(\exp \left(-2 \underline{r}_{t}\right)\right) .
$$




\section{Existence and Uniqueness of Constant Mean Curvature Foliations}

8.1 Uniqueness. We are now ready to prove the main theorem.

Theorem 8.1. If the mass of the asymptotically anti-de Sitter-Schwarzschild metric is nonzero, then any two smooth foliations by stable spheres with constant mean curvature $\left(\Sigma_{t}^{1}\right)_{t \geq 0}$ and $\left(\Sigma_{t}^{2}\right)_{t \geq 0}$ for which

$$
\lim _{t \rightarrow \infty}\left(\bar{r}_{t}-4 / 3 \underline{r}_{t}\right)=-\infty
$$

will coincide for $t$ sufficiently large.

Proof. We can reparametrize the foliations so that $H\left(\Sigma_{t}^{1}\right)=H\left(\Sigma_{t}^{2}\right)=H_{t}$ for all $t$ sufficiently large. The results in the previous section imply the existence of $\tilde{r}_{t}$ so that

$$
H_{t}=2 \cosh \tilde{r}_{t} / \sinh \tilde{r}_{t}-m / \sinh ^{3} \tilde{r}_{t}+o\left(\exp \left(-4 \tilde{r}_{t}\right)\right),
$$

and, for $i=1,2$, the functions

$$
w_{t}^{i} \equiv r(x)-\tilde{r}_{t}
$$

have $C^{2, \alpha}$ norm of order $o\left(\exp \left(-\underline{r}_{t}\right)\right)$, where the norm is computed with respect to the rescaled metric $\hat{g}_{t}^{i}$. From Theorem 5.1 we know that, after pulling back $\hat{g}_{t}^{1}$ by a suitable diffeomorphism, the metric can be written as $\exp \left(2 \beta_{t}\right) g_{0}$, where $g_{0}$ denotes the standard round metric on $S^{2}$. The following lemma improves the estimate on $\beta_{t}$.

LEMma 8.2. The functions $\beta_{t}$ can be chosen so that

$$
\left|\beta_{t}\right|_{C^{2, \alpha}}=o\left(\exp \left(-\underline{r}_{t}\right)\right) \text {. }
$$

Proof. A direct computation using the fact that each $\Sigma_{t}^{1}$ is the graph over $\left\{|x|=\tilde{r}_{t}\right\}$ of a function $w_{t}^{1}$ with $\left|w_{t}^{1}\right|_{C^{2, \alpha}}=o\left(\exp \left(-\underline{r}_{t}\right)\right)$, reveals that $|\stackrel{\AA}{A}|_{C^{0, \alpha}}^{2}=o\left(\exp \left(-2 \underline{r}_{t}\right)\right)$, where the norm is computed with respect to the metric $g_{0}$. Combining Lemma 3.2 with the asymptotic expansion of $H_{t}$ in terms of $\tilde{r}_{t}$, we obtain that the Gaussian curvature of $\hat{g}_{t}^{1}$ can be written as

$$
\widehat{K}_{t}=1+S_{t}, \quad \text { where } \quad\left|S_{t}\right|_{C^{0, \alpha}}=o\left(\exp \left(-\underline{r}_{t}\right)\right) .
$$

The functions $\beta_{t}$ were chosen so that, for each coordinate function $x_{1}, x_{2}$, and $x_{3}$,

$$
\int_{S^{2}} \exp \left(2 \beta_{t}\right) x_{i} d \mu_{0}=0, \quad i=1,2,3,
$$

and they satisfy the equation

$$
\begin{aligned}
\Delta_{0} \beta_{t} & =1-\widehat{K}_{t} \exp \left(2 \beta_{t}\right) \\
& =1-\exp \left(2 \beta_{t}\right)+\exp \left(2 \beta_{t}\right) o(\exp (-r)) .
\end{aligned}
$$

One can then use the smallness of $\beta_{t}$ and argue in the same way as in the proof of either Theorem 5.1 or Theorem 7.1 in order to prove the lemma.

With respect to the coordinates $(r, \omega)$ in $\mathbb{R} \times S^{2}$ we consider, for each fixed $t$, the interpolation surfaces

$$
\Sigma_{t, s}=\left\{\tilde{r}_{t}+(1-s) w_{t}^{1}(\omega)+s w_{t}^{2}(\omega) \mid \omega \text { in } S^{2}\right\}, \quad 1 \leq s \leq 0,
$$

and set

$$
F_{t}(s) \equiv H\left(\Sigma_{t, s}\right) .
$$


In what follows, we fix $t$ large and suppress the index $t$ in the notation for the sake of simplicity. From Taylor's formula, we have

where

$$
F(1)=F(0)+F^{\prime}(0)+P, \quad P \equiv \int_{0}^{1} \int_{0}^{1} s F^{\prime \prime}(s u) d u d s
$$

$$
F^{\prime}(0)=\Delta_{\Sigma_{1}} \phi+\left(|A|^{2}+R(\nu, \nu)\right)_{\Sigma_{1}} \phi, \quad \phi \equiv w^{2}-w^{1},
$$

and, for some universal constant $C$,

$$
\left|F^{\prime \prime}(s)\right| \leq C\left(\left|\nabla^{2} \phi\right||\phi|+|\nabla \phi|^{2}+|\phi|^{2} \exp (-2 r)\right)_{\mid \Sigma_{t, s}} .
$$

We know that $F(1)=F(0)$ and that

$$
\begin{aligned}
\left(|A|^{2}+R(\nu, \nu)\right)_{1} & =\left(H^{2}-4\right) / 2-m / \sinh ^{3} \tilde{r}+O(\exp (-4 r)) \\
& =8 \pi /\left|\Sigma^{1}\right|-3 m / \sinh ^{3} \tilde{r}+O(\exp (-4 r)) .
\end{aligned}
$$

Therefore, using Theorem 7.1, we have that, with respect to the metric $g_{0}, \phi$ satisfies the following equation

$$
\Delta_{0} \phi+\exp (2 \beta)(2-3 m / \sinh \tilde{r}+O(\exp (-2 r))) \phi+\left|\Sigma^{1}\right|(4 \pi)^{-1} \exp (2 \beta) P=0,
$$

or, alternatively,

$$
\Delta_{0} \phi+(2-3 m / \sinh \tilde{r}) \phi=Q-\left|\Sigma^{1}\right|(4 \pi)^{-1} \exp (2 \beta) P,
$$

where

$$
Q \equiv(1-\exp (2 \beta))(2-3 m / \sinh \tilde{r}) \phi-\exp (2 \beta) O(\exp (-2 r)) \phi
$$

Consider the decomposition

$$
\phi=\phi_{0}+\phi_{1}, \quad Q=Q_{0}+Q_{1}, \quad\left|\Sigma^{1}\right| \exp (2 \beta) P=P_{0}+P_{1},
$$

where $\phi_{0}, Q_{0}, P_{0}$ belong to the kernel of $\Delta_{0}+2$ and $\phi_{1}, Q_{1}, P_{1}$ are orthogonal to $\phi_{0}, Q_{0}, P_{0}$ respectively. Then

$$
\begin{gathered}
\Delta_{0} \phi_{1}+(2-3 m / \sinh \tilde{r}) \phi_{1}=Q_{1}-P_{1}, \\
3 m / \sinh \tilde{r} \phi_{0}=P_{0}-Q_{0},
\end{gathered}
$$

and it is immediate to recognize that, for some universal constant $C$,

$$
\left|P_{0}\right|_{C^{0, \alpha}}+\left|P_{1}\right|_{C^{0, \alpha}} \leq C|\phi|_{C^{2, \alpha}}^{2}
$$

and

$$
\left|Q_{0}\right|_{C^{0, \alpha}}+\left|Q_{1}\right|_{C^{0, \alpha}} \leq o(\exp (-\underline{r}))|\phi|_{C^{0, \alpha}},
$$

where the norms are computed with respect to $g_{0}$.

Applying Schauder estimates to equation (26) and using the fact that $|\phi|_{C^{2, \alpha}}$ converges to zero, we obtain

$$
\left|\phi_{1}\right|_{C^{2, \alpha}} \leq C \sup _{\Sigma^{1}}\left|\phi_{1}\right|+C\left|\phi_{0}\right|_{C^{2, \alpha}}^{2}+o(\exp (-\underline{r}))\left|\phi_{0}\right|_{C^{0, \alpha}}
$$

where $C$ is some uniform constant. The orthogonality condition satisfied by $\phi_{1}$ can be used in the same way as in the proof of Theorem 7.1 in order to show that

Therefore,

$$
\sup _{\Sigma^{1}}\left|\phi_{1}\right| \leq C|\phi|_{C^{2, \alpha}}^{2}+o(\exp (-\underline{r}))|\phi|_{C^{0, \alpha}} .
$$

$$
\left|\phi_{1}\right|_{C^{2, \alpha}} \leq C\left|\phi_{0}\right|_{C^{2, \alpha}}^{2}+o(\exp (-\underline{r}))\left|\phi_{0}\right|_{C^{0, \alpha}},
$$


where $C$ is some uniform constant. Because the $C^{2, \alpha}$ norm of $\phi_{0}$ is bounded by its $C^{0, \alpha}$ norm, we obtain from $(27)$ that

$$
\begin{aligned}
\left|\phi_{0}\right|_{C^{2, \alpha}} & \leq O(\exp (\underline{r}))|\phi|_{C^{2, \alpha}}^{2}+o(1)|\phi|_{C^{0, \alpha}} \\
& \leq O(\exp (\underline{r}))\left|\phi_{0}\right|_{C^{2, \alpha}}^{2}+o(1)\left|\phi_{0}\right|_{C^{0, \alpha}} .
\end{aligned}
$$

From Theorem 7.1 we have that $|\phi|_{C^{2, \alpha}}=o(\exp (-\underline{r}))$ and thus

$$
\left|\phi_{0}\right|_{C^{0, \alpha}} \leq o(1)\left|\phi_{0}\right|_{C^{0, \alpha}} \text {. }
$$

Consequently, for $t$ sufficiently large, $\phi_{0}=0$ and this implies that $\phi_{1}=0$. Hence $w_{t}^{1}=w_{t}^{2}$ for all $t$ sufficiently large and this is the same as $\Sigma_{t}^{1}=\Sigma_{t}^{2}$.

8.2 Existence. We show existence of a foliation by stable spheres with constant mean curvature when the mass of the asymptotically anti-de Sitter-Schwarzschild metric is positive. This result was previously shown by Rigger [R] using a modified mean curvature flow approach. The argument we use is a straightforward adaptation of the arguments used by Rugang Ye in [Y], where he showed a similar theorem in the context of asymptotically flat manifolds. We include the proof of existence of a foliation for the sake of completeness.

Theorem 8.3. If the mass of the asymptotically anti-de Sitter-Schwarzschild metric is nonzero, the manifold $M$ admits, outside a compact set, a foliation by spheres with constant mean curvature. Moreover, if the mass is positive, then the spheres are stable.

Proof. Given $\phi \in C^{\infty}\left(S^{2}\right)$ and $r$ sufficiently large, let

$$
\Sigma_{r}(\phi) \equiv\left\{(r+\phi(x), x) \mid x \in S^{2}\right\},
$$

and set $F(r, \phi) \equiv H\left(\Sigma_{r}(\phi)\right)$. Because the metric $g$ is a $C^{3}$ perturbation of $g_{m}$, we have from Lemma 3.1 that

$$
F(r, 0)=2 \cosh r / \sinh r-m / \sinh ^{3} r+O(\exp (-5 r))
$$

and

$$
\left(|A|^{2}+R(\nu, \nu)\right)_{\Sigma_{r}(0)}=8 \pi /\left|\Sigma_{r}(0)\right|-3 m / \sinh ^{3} r+O(\exp (-5 r)) .
$$

For every $r$ sufficiently large, we want to find $\phi$ so that

$$
F(r, \phi)=2 \cosh r / \sinh r-m / \sinh ^{3} r .
$$

Using Taylor's formula like in the previous subsection, the above equation is equivalent to solve on $S^{2}$

$$
\Delta_{0} \phi+(2-3 m / \sinh r) \phi=P(\phi)+Q(\phi)+N,
$$

where

$$
N \equiv\left|\Sigma_{r}(0)\right|\left(F(r, 0)-2 \cosh r / \sinh r+m / \sinh ^{3} r\right) /(4 \pi)
$$

satisfies

$$
|N|_{C^{0, \alpha}}=O(\exp (-3 r))
$$

and $P$ and $Q$ are such that, for some uniform constant $C$,

$$
\begin{gathered}
|P(\phi)|_{C^{0, \alpha}} \leq C|\phi|_{C^{2, \alpha}}^{2} \\
|P(\phi)-P(\psi)|_{C^{0, \alpha}} \leq C\left(|\phi|_{C^{2, \alpha}}+|\psi|_{C^{2, \alpha}}\right)|\phi-\psi|_{C^{2, \alpha}}, \\
|Q(\phi)|_{C^{0, \alpha}} \leq o(\exp (-r))|\phi|_{C^{0, \alpha}}
\end{gathered}
$$


and

$$
|Q(\phi)-Q(\psi)|_{C^{0, \alpha}} \leq o(\exp (-r))|\phi-\psi|_{C^{0, \alpha}}
$$

Consider the map

$$
T: C^{2, \alpha}\left(S^{2}\right) \longrightarrow C^{2, \alpha}\left(S^{2}\right)
$$

such that

$$
\Delta_{0}(T(u))+(2-3 m / \sinh r) T(u)=P(u)+Q(u)+N .
$$

The map is well defined because the operator on the left hand side is invertible. The existence of a constant mean curvature foliation follows from

LEMmA 8.4. For all $r$ sufficiently large, the map $T$ is a contraction of

onto itself.

$$
\left\{\left.u \in C^{2, \alpha}\left(S^{2}\right)|| u\right|_{C^{2, \alpha}} \leq \exp (-r) / r\right\}
$$

Proof. Set $\phi \equiv T(u)$ and consider the decomposition

$$
\phi=\phi_{0}+\phi_{1}, \quad P(u)=P_{0}+P_{1}, \quad Q(u)=Q_{0}+Q_{1}, \quad \text { and } N=N_{0}+N_{1},
$$

such that $\phi_{0}, P_{0}, Q_{0}, N_{0}$ belong to the kernel of $\Delta+2$ and $\phi_{1}, P_{1}, Q_{1}, N_{1}$ are orthogonal to $\phi_{0}, P_{0}, Q_{0}, N_{0}$ respectively. Thus,

$$
-3 m \phi_{0} / \sinh r=P_{0}+Q_{0}+N_{0}
$$

and so

$$
\left|\phi_{0}\right|_{C^{2, \alpha}} \leq O(\exp (r))|u|_{C^{2, \alpha}}^{2}+o(1)|u|_{C^{2, \alpha}}+O(\exp (-2 r)) \leq o(1) \exp (-r) / r .
$$

Furthermore,

$$
\Delta_{0} \phi_{1}+(2-3 m / \sinh r) \phi_{1}=P_{1}(u)+Q_{1}(u)+N_{1}
$$

and hence, we can argue in the same way as in the proof of Theorem 5.1 and use the orthogonality condition satisfied by $\phi_{1}$ in order to show that

$$
\left|\phi_{1}\right|_{C^{2, \alpha}} \leq C|u|_{C^{2, \alpha}}^{2}+o(\exp (-r))|u|_{C^{2, \alpha}}+O(\exp (-3 r)) \leq o(1) \exp (-r) / r
$$

for some uniform constant $C$. Therefore, we have for all $r$ sufficiently large that $|\phi|_{C^{2, \alpha}} \leq \exp (-r) / r$. Finally, we can argue in the same way and check that

$$
|T(u)-T(v)|_{C^{2, \alpha}} \leq o(1)|u-v|_{C^{2, \alpha}} .
$$

Denote by $\Sigma_{r}$ the constant mean curvature sphere that is the graph over $\{|x|=r\}$ of a function $w_{r}$ with $\left|w_{r}\right|_{C^{2, \alpha}} \leq \exp (-r) / r$, where the norm is computed with respect to the standard round metric $g_{0}$. We need to show that $\Sigma_{r}$ is stable for all $r$ sufficiently large, i.e. we need to show that second variation operator

$$
L f \equiv-\Delta f-\left(|A|^{2}+R(\nu, \nu)\right) f
$$

has only nonnegative eigenvalues when restricted to functions with zero mean value.

Because $\Sigma_{r}$ is the graph of a function with $\left|w_{r}\right|_{C^{2, \alpha}} \leq o(\exp (-r))$, we have that

$$
\sup _{\Sigma_{r}}|A|^{2}=o(\exp (-4 r)),
$$

where this norm is computed with respect to the metric $g_{r}$ induced by the ambient metric. Moreover, we also have that

$$
|A|^{2}+R(\nu, \nu)=8 \pi /\left|\Sigma_{r}\right|-3 m / \sinh ^{3} r+O(\exp (-4 r)) .
$$

In this setting, Theorem 5.1 and Lemma 8.2 apply and so, after applying a suitable diffeomorphism, the normalized metric $\hat{g}_{r} \equiv 4 \pi /\left|\Sigma_{r}\right| g_{r}$ can be written as $\exp \left(2 \beta_{r}\right) g_{0}$, 
where $\left|\beta_{r}\right|_{C^{2, \alpha}} \leq o(\exp (-r))$. Hence, in terms of the metric $g_{0}$, the positivity of the operator $L$ is equivalent to the positivity of the following operator defined on $S^{2}$

$$
L_{0} \phi \equiv-\Delta_{0} \phi-(2-3 m / \sinh r+o(\exp (-r)) \phi) .
$$

This is true for all $r$ sufficiently large whenever $m$ is positive.

\section{Appendix}

\section{A Proof of Lemma 6.5}

Given a smooth function $u$ on $S^{2}$, recall that

$$
S[u] \equiv \int_{S^{2}}\left|\nabla_{0} u\right|^{2} d \mu_{0}-2 \int_{S^{2}} u d \mu_{0} .
$$

We want to show that $S\left[w_{t}\right]$ is bounded independently of $t$. We essentially follow, with some necessary modifications, the proof of [CY, Prop.4.1]. The main idea consists in exploiting the invariance of $S$ under conformal transformations. More precisely, we will find conformal diffeomorphisms $F_{t}$ for which the family of functions $u_{t}$ defined by

is such that

$$
\exp \left(-2 u_{t}\right) g_{0}=F_{t}^{*}\left(\exp \left(-2 w_{t}\right) g_{0}\right)
$$

$$
\int_{S^{2}}\left|\nabla u_{t}\right|^{2} d \mu_{0} \quad \text { and } \quad \int_{S^{2}} u_{t} d \mu_{0}
$$

are uniformly bounded. The desired result follows because $S\left[u_{t}\right]=S\left[w_{t}\right]$.

A standard application of Brower's fixed point theorem (see for instance [C1, Lect. 3, Lem.2]) implies the existence of a conformal diffeomorphism $T$ such that, for $i=1,2,3$,

$$
\int_{S^{2}} x_{i} \exp \left(-4 w_{t} \circ F_{t}+4 \gamma_{t}\right) d \mu_{0}=0, \quad \text { where } F_{t}^{*}\left(g_{0}\right)=\exp \left(2 \gamma_{t}\right) g_{0} \text {. }
$$

Moreover, $\gamma_{t}$ has an upper bound given by

Lemma A.1. There is a universal constant $C_{0}$ so that

$$
\sup \left|\gamma_{t}\right| \leq C_{0}+2 / 3\left(\bar{r}_{t}-\underline{r}_{t}\right) \text {. }
$$

Proof. Assume that the maximum of $\alpha_{t}$ is attained at the north pole $p$ in $S^{2}$. We know that

$$
\int_{\left\{x_{3} \geq 0\right\}} x_{3} \exp \left(-4 w_{t} \circ T+4 \gamma_{t}\right) d \mu_{0}=\int_{\left\{x_{3} \leq 0\right\}}\left(-x_{3}\right) \exp \left(-4 w_{t} \circ T+4 \gamma_{t}\right) d \mu_{0}
$$

and so

$$
1 / 2 \int_{\left\{x_{3} \geq 1 / 2\right\}} \exp \left(-4 \sup w_{t}+4 \gamma_{t}\right) d \mu_{0} \leq \int_{\left\{x_{3} \leq 0\right\}} \exp \left(-4 \inf w_{t}+4 \gamma_{t}\right) d \mu_{0} .
$$

In stereographic coordinates, $\gamma_{t}$ can be written as

$$
\gamma_{t}(x)=\log \left(\lambda\left(1+|x|^{2}\right) /\left(\lambda^{2}+|x|^{2}\right)\right)
$$

for some $\lambda \leq 1$. Note that $\sup \gamma_{t}=-\inf \gamma_{t}=-\log \lambda$. An explicit computation shows the existence of some $r_{0}$ so that, if

$$
f(r) \equiv 8 / 3 \lambda^{4}\left(\lambda^{4}+\lambda^{2}+3 r^{4}+3\left(\lambda^{2}+1\right) r^{2}+1\right) /\left(\lambda^{2}+r^{2}\right)^{3},
$$


then

and

$$
\int_{\left\{x_{3} \geq 1 / 2\right\}} \exp \left(4 \gamma_{t}\right) d \mu_{0}=f(0)-f\left(r_{0}\right)
$$

$$
\int_{\left\{x_{3} \leq 0\right\}} \exp \left(4 \gamma_{t}\right) d \mu_{0}=f(1) .
$$

Hence, there are universal constants $C_{1}, C_{2}$, and $C_{3}$ so that, for all $\lambda \leq 1$,

and

$$
\int_{\left\{x_{3} \geq 1 / 2\right\}} \exp \left(4 \gamma_{t}\right) d \mu_{0} \geq C_{1} \lambda^{-2}-C_{2} \lambda^{4}
$$

$$
\int_{\left\{x_{3} \leq 0\right\}} \exp \left(4 \gamma_{t}\right) d \mu_{0} \leq C_{3} \lambda^{4} .
$$

Therefore, (29) implies that, for some universal constants $C_{5}$

$$
\lambda^{-6} \leq C_{5} \exp \left(4 \sup w_{t}-4 \inf w_{t}\right)+C_{5} .
$$

The result follows because $\sup w_{t}-\inf w_{t}=\bar{r}_{t}-\underline{r}_{t}$.

Let

$$
u_{t} \equiv w_{t} \circ T-\gamma_{t}+\log \delta_{t} / 2, \quad \text { where } \delta_{t} \equiv f_{S^{2}} \exp \left(-2 w_{t}\right) d \mu_{0} .
$$

The effect of $\delta_{t}$ is to ensure that $\exp \left(-2 u_{t}\right) g_{0}$ has volume $4 \pi$ and we observe that, combining Theorem 5.1 with Proposition 4.2, we obtain

$$
\delta_{t}=1+O\left(\exp \left(-\underline{r}_{t}\right)\right)+O\left(\exp \left(2 \bar{r}_{t}-3 \underline{r}_{t}\right)\right) .
$$

Because

$$
S\left[u_{t}\right]=S\left[w_{t}\right]-\log \delta_{t},
$$

it suffices to show that $S\left[u_{t}\right]$ is uniformly bounded.

Because

$$
\Delta_{0} \gamma_{t}=1-\exp \left(2 \gamma_{t}\right)
$$

we have (14) that

$$
\Delta_{0} u_{t}=\exp \left(-2 u_{t}\right)-1+Q_{t}
$$

where

$$
Q_{t}=\exp \left(2 \gamma_{t}\right) P_{t} \circ T+\exp \left(-2 u_{t}\right)\left(\delta_{t}-1\right) .
$$

Integration by parts yields

$$
2 \int_{S^{2}}\left|\nabla_{0} u_{t}\right|^{2} d \mu_{0}-2 \int_{S^{2}} u_{t} d \mu_{0}=\int_{S^{2}}-2 u_{t} \exp \left(-2 u_{t}\right) d \mu_{0}-\int_{S^{2}} 2 u_{t} Q_{t} d \mu_{0} .
$$

We argue that the last term on the right-hand side is bounded independently of $t$. Note that Lemma 6.2 and Lemma A.1 imply that

$$
\begin{aligned}
\int_{S^{2}}\left|u_{t} Q_{t}\right| d \mu_{0} & \leq \int_{S^{2}}\left|u_{t} \circ T^{-1} P_{t}\right| d \mu_{0}+\left|\delta_{t}-1\right| \int_{S^{2}}\left|u_{t} \circ T^{-1}\right| \exp \left(-2 w_{t}\right) d \mu_{0} \\
& \leq\left(\left|\bar{r}_{t}-\underline{r}_{t}\right|+C_{0}\right)\left(O\left(-\underline{r}_{t}\right)+O\left(\exp \left(2 \bar{r}_{t}-3 \underline{r}_{t}\right)\right)\right),
\end{aligned}
$$

and so, because we are assuming condition (1), we obtain

$$
\lim _{t \rightarrow \infty} \int_{S^{2}}\left|u_{t} Q_{t}\right| d \mu_{0}=0 .
$$

Thus, using Jensen's inequality we obtain that, for all $t$ sufficiently large,

$$
2 \int_{S^{2}}\left|\nabla_{0} u_{t}\right|^{2} d \mu_{0}-2 \int_{S^{2}} u_{t} d \mu_{0} \leq 4 \pi \log f_{S^{2}} \exp \left(-4 u_{t}\right) d \mu_{0}+1 .
$$


LEMMA A.2.

$$
\int_{S^{2}}\left|\nabla_{0} u_{t}\right| d \mu_{0}
$$

is uniformly bounded independently of $t$.

Proof. Denoting by $G(x, y)$ the Green's function for the Laplacian on $S^{2}$ we have

$$
u_{t}(x)=\int_{S^{2}} u_{t} d \mu_{0}-\int_{S^{2}} \Delta_{0} u_{t}(y) G(x, y) d \mu_{0}(y),
$$

and so, because $\nabla G(\cdot, y)$ is in $L^{1}$ for all $y$,

for some constant $C$ independent of $t$.

$$
\int_{S^{2}}\left|\nabla_{0} u_{t}\right| d \mu_{0} \leq \int_{S^{2}}\left|\Delta_{0} u_{t}\right| d \mu_{0} \leq C
$$

This lemma and identity (28) allow us to use an improvement of Aubin's inequality $[\mathrm{A}]$, due to Alice Chang and Paul Yang [CY, Lemma 4.2 ], which says that under these conditions

$$
4 \pi \log f_{S^{2}} \exp \left(-4 u_{t}\right) d \mu_{0} \leq 2 \int_{S^{2}}\left|\nabla_{0} u_{t}\right|^{2} d \mu_{0}-4 \int_{S^{2}} u_{t} d \mu_{0}+C,
$$

where $C$ is a constant independent of $t$. Combining this with (30) we get that

$$
\hat{u}_{t} \equiv \int_{S^{2}} u_{t} d \mu_{0}
$$

is uniformly bounded from above. A uniform bound below follows trivially from $\exp \left(-2 u_{t}\right) g_{0}$ having volume $4 \pi$. In order to bound the gradient term we integrate by parts again so that, for all $t$ sufficiently large,

$$
\begin{aligned}
2 \int_{S^{2}}\left|\nabla_{0} u_{t}\right|^{2} d \mu_{0}= & 2 \int_{S^{2}} \exp \left(-2 u_{t}\right)\left(\hat{u}_{t}-u_{t}\right) d \mu_{0}+\int_{S^{2}} 2\left(\hat{u}_{t}-u_{t}\right) Q_{t} d \mu_{0} \\
\leq & 2 \int_{S^{2}}\left(\exp \left(-2 u_{t}\right)-2 \pi\right)\left(\hat{u}_{t}-u_{t}\right) d \mu_{0}+1 \\
\leq & 2 \pi \log \left((2 \pi)^{-1} \int_{S^{2}}\left(\exp \left(-2 u_{t}\right)-2 \pi\right) \exp \left(2\left(\hat{u}_{t}-u_{t}\right)\right) d \mu_{0}\right) \\
& +1 \\
\leq & 2 \pi \log \int_{S^{2}} \exp \left(-4 u_{t}\right) d \mu_{0}+C \\
\leq & \int_{S^{2}}\left|\nabla_{0} u_{t}\right|^{2} d \mu_{0}+C,
\end{aligned}
$$

where $C$ is a constant independent of $t$.

\section{References}

[A] T. Aubin, Meilleures constantes dans le théorème d'inclusion de Sobolev et un théorème de Fredholm non linéaire pour la transformation conforme de la courbure scalaire, J. Funct. Anal. 32 (1979), 148-174.

[C1] S.-Y.A. Chang, The Moser-Trudinger inequality and applications to some problems in conformal geometry, in "Nonlinear Partial Differential Equations in Differential Geometry (Park City, UT, 1992), IAS/Park City Math. Ser. 2, Amer. Math. Soc. Providence, RI (1996). 
[C2] S.-Y.A. Chang, Non-linear Elliptic Equations in Conformal Geometry, Zurich Lectures in Advanced Mathematics, European Mathematical Society (EMS), Zurich, 2004.

[CY] S.-Y.A. Chang, P. YAng, A perturbation result in prescribing scalar curvature on $S^{n}$, Duke Math. J. 64:1 (1991), 27-69.

[ChY] D. Christodoulou, S.-T. Yau, Some remarks on the quasi-local mass, Mathematics and General Relativity (Santa Cruz, CA, 1986), Contemp. Math. 71, Amer. Math. Soc., Providence, RI (1988), 9-14.

[ChrH] P. Chruściel, M. Herzlich, The mass of asymptotically hyperbolic Riemannian manifolds, Pacific J. Math. 212 (2003), 231-264.

[CoM] T. Colding, W. Minicozzi, Minimal Surfaces, Courant Lecture Notes in Mathematics, New York University, Courant Institute of Mathematical Sciences, New York, 1999.

[H] D. Hoffman, J. Spruck, Sobolev and isoperimetric inequalities for Riemannian submanifolds, Comm. Pure Appl. Math. 27 (1974), 715-727.

[HuI $]$ G. Huisken, T. Ilmanen, The inverse mean curvature flow and the Riemannian Penrose inequality, J. Differential Geom. 59 (2001), 353-437.

[HuP] G. Huisken, A. Polden, Geometric evolution equations for hypersurfaces, Calculus of Variations and Geometric Evolution Problems (Cetraro, 1996), Springer Lecture Notes in Math. 1713(1999), 45-84.

[HuY] G. Huisken, S.-T. YAu, Definition of center of mass for isolated physical systems and unique foliations by stable spheres with constant mean curvature, Invent. Math. 124 (1996), 281-311.

[K] J. KAZDAN, F. WARner, Existence and conformal deformation of metrics with prescribed Gaussian and scalar curvatures, Ann. of Math. (2) 101 (1975), 317-331.

[L] P. LI, Lecture Notes on Geometric Analysis, Lecture Notes Series 6, Seoul National University, Research Institute of Mathematics, Global Analysis Research Center, Seoul, 1993.

[QT] J. QING, G. TIAN, On the uniqueness of the foliation of spheres of constant mean curvature in asymptotically flat 3-manifolds, preprint.

[R] R. RigGeR, The foliation of asymptotically hyperbolic manifolds by surfaces of constant mean curvature (including the evolution equations and estimates), Manuscripta Math. 113 (2004), 403-421.

[T] N. Trudinger, On imbeddings into Orlicz spaces and some applications, J. Math. Mech. 17 (1967), 473-483.

[W] X. WANG, The mass of asymptotically hyperbolic manifolds, J. Differential Geom. 57 (2001), 273-299.

[Y] R. YE, Foliation by constant mean curvature spheres on asymptotically flat manifolds, Geometric Analysis and the Calculus of Variations, Int. Press, Cambridge, MA (1996), 369-383.

André Neves, Fine Hall, Princeton University, Princeton, NJ 08544, USA aneves@math.princeton.edu

Gang Tian, Fine Hall, Princeton University, Princeton, NJ 08544, USA

tian@math.princeton.edu

Received: February 21, 2008

Accepted: July 27, 2008

Open Access This article is distributed under the terms of the Creative Commons Attribution Noncommercial License which permits any noncommercial use, distribution, and reproduction in any medium, provided the original author(s) and source are credited. 